\title{
Der grenzüberschreitende Sachverhalt - Der Yeti des Europarechts
}

\author{
André Lippert*
}

Inhalt

A. Einleitung 274

B. Der grenzüberschreitende Sachverhalt im Unionsrecht als quantitatives Kriterium $\quad 274$

I. Der grenzüberschreitende Sachverhalt in der Rechtsprechung des EuGH zu den Grundfreiheiten

1. Das Negativkriterium „kein rein interner Sachverhalt“

2. Das Positivkriterium „Auswirkungen auf den innergemeinschaftlichen Handel“"

3. Die Weite des EuGH-Kriteriums 278

a) Der Grenzübertritt in Vergangenheit und Zukunft 279

(1) Rückkehrerfälle 279

(2) Wegzugsfälle 279

b) Der hypothetische Grenzübertritt 280

c) Der wirtschaftliche Vergleichsmaßstab 281

4. Das Ziel: Unionsweite Mobilität -

Das Mittel: Der ökonomisch relevante Sachverhalt 282

a) Das Ziel des EuGH: Unionsweite Mobilität 282

b) Die Vermutungsregel 283

II. Der grenzüberschreitende Sachverhalt in der Rechtsprechung des EuGH zum Wettbewerbsrecht 285

III. Quantitative Kriterien und Subsidiarität am Beispiel der Fusionskontrolle 287

1. Die Aufgreifschwelle in der Fusionskontrolle als quantitatives Kriterium 288

2. Subsidiaritätsprinzip, Kontrollkompetenzen und grenzüberschreitender Sachverhalt 288

a) Der transnationale Bezug im Subsidiaritätsprinzip 288

b) Kontrollkompetenzen 290

c) Der grenzüberschreitende Sachverhalt bei den Kontrollkompetenzen 293

* Der Verfasser ist Rechtsanwalt im Berliner Büro der überörtlichen Sozietät Gleiss Lutz. Er wurde bei Prof. Dr. Christian Calliess, Lehrstuhl für Öffentliches Recht und Europarecht an der Freien Universität Berlin mit dem Thema „Der grenzüberschreitende Sachverhalt im Unionsrecht“ (Tübingen 2013) promoviert. 
C. Die Alternative: Ein qualitatives Kriterium 294

I. Ein rechtlicher Maßstab 294

II. Eine neue Definition des grenzüberschreitenden Sachverhalts 296

D. Fazit: Die Bedeutung des grenzüberschreitenden Sachverhalts im Unionsrecht 297

\section{A. Einleitung}

Der grenzüberschreitende Sachverhalt ist der Yeti des Unionsrechts: Jeder kennt ihn, aber keiner hat ihn bislang wirklich gesehen. Dies muss erstaunen, scheinen doch Rechtsprechung, Literatur und Praxis einhellig davon auszugehen, dass das Kriterium unverzichtbar und eines der zentralen Prinzipien des gesamten Unionsrechts ist. Gleichwohl findet sich - soweit ersichtlich - nirgends der Versuch einer inhaltlichen Umschreibung oder gar Definition. ${ }^{1}$ Vor diesem Hintergrund unternimmt der Autor den Versuch einer Annäherung an den grenzüberschreitenden Sachverhalt als Merkmal und Kategorie des Unionsrechts auf der Grundlage der Rechtsprechung des Europäischen Gerichtshofs (EuGH). Der Untersuchungsgegenstand beschränkt sich dabei auf die so genannten Kontrollkompetenzen, eine Kompetenzkategorie, die hier in Abgrenzung zu den Legislativkompetenzen der Union verwendet wird. Am Ende steht der Versuch einer Definition, die der Bedeutung des grenzüberschreitenden Elements als notwendiges Strukturmerkmal und Schlüsselprinzip der gesamten Unionsrechtsordnung Rechnung trägt und dabei vor allem Funktion und Aufgabe dieses Merkmals im Mehrebenensystem Europäische Union berücksichtigt.

\section{B. Der grenzüberschreitende Sachverhalt im Unionsrecht als quantitatives Kriterium}

Im Primärrecht wird der grenzüberschreitende Sachverhalt nur indirekt erwähnt. In der Präambel beispielsweise ist nur davon die Rede, dass die Mitgliedstaaten

„entschlossen [sind], durch gemeinsames Handeln den wirtschaftlichen und sozialen Fortschritt ihrer Länder zu sichern, indem sie die Europa trennenden Schranken beseitigen [...]. [Sie handeln] in dem Wunsch, durch eine gemeinsame Handelspolitik zur fortschreitenden Beseitigung der Beschränkungen im zwischenstaatlichen Wirtschaftsverkehr beizutragen“. ${ }^{2}$

Bei den Grundfreiheiten, die zu den wichtigsten und wohl auch schlagkräftigsten Instrumenten der Union zählen, findet häufig der Terminus ,zwischen den Mitgliedstaaten“ Verwendung. Dies gilt beispielsweise für die Maßnahmen gleicher Wirkung wie Ein- und Ausfuhrbeschränkungen (Art. 34, 35 AEUV). Es muss sich also offensichtlich um eine

1 In der Literatur widmen sich - soweit ersichtlich - nur zwei Veröffentlichungen ausdrücklich dem grenzüberschreitenden Sachverhalt als eigenständiges Prinzip, und zwar Papadileris, Das Erfordernis des grenzüberschreitenden Bezugs im Recht der Marktfreiheiten, JuS 2011, S. 123 und Kutzscher, Der grenzüberschreitende Sachverhalt in der Rechtsprechung des EuGH und deren Auswirkungen auf die Freizügigkeit der Unionsbürger, 2011.

2 Präambel AEUV; Präambel EG-Vertrag. 
Maßnahme handeln, die den tatsächlich zwischen (zwei oder mehreren) Mitgliedstaaten bestehenden Handelsverkehr beeinträchtigt.

Auch Art. 49 AEUV verbietet „Beschränkungen der freien Niederlassung von Staatsangehörigen eines Mitgliedstaats im Hoheitsgebiet eines anderen Mitgliedstaats“, womit zwischen Herkunfts- und Zielmitgliedstaat differenziert wird. ${ }^{3}$

Mit der Staatsangehörigkeit wird damit ein zweites Kriterium genannt, das vor allem bei den Grundfreiheiten, die nicht an ein Produkt, sondern an eine Person anknüpfen, eine Rolle spielt. So umfasst nach Art. 45 Abs. 2 AEUV die Arbeitnehmerfreizügigkeit die Abschaffung ,jeder auf der Staatsangehörigkeit beruhenden unterschiedlichen Behandlung der Arbeitnehmer der Mitgliedstaaten“.

Das entspricht im Übrigen auch dem allen Grundfreiheiten als Grundnorm zugrundliegenden Diskriminierungsverbot des Art. 18 AEUV, das ausdrücklich auf eine Diskriminierung aus Gründen der Staatsangehörigkeit abstellt. Diese Norm ist Leitmotiv und materieller Kern der Verträge, ${ }^{4}$ zielt sie doch auf die Überwindung des Fremdenstatus und die Gleichbehandlung eines jeden Unionsbürgers in jedem Mitgliedstaat. ${ }^{5}$ Mit ihr wird die Grundlage des Binnenmarktes beschrieben, da die Diskriminierung aufgrund der Staatsangehörigkeit die offensichtlichste Form der nicht erwünschten Grenzerrichtung auf dem Binnenmarkt ist. ${ }^{6}$

Im Kartell- und Beihilfenrecht (Art. 101, 102, 107 AEUV) schließlich findet sich in allen wichtigen Normen des Primärrechts die so genannte Zwischenstaatlichkeitsklausel, nach der eine bestimmte Verhaltensweise dann verboten ist, wenn diese den „Handel zwischen den Mitgliedstaaten“ beeinträchtigen kann.

\section{Der grenzüberschreitende Sachverhalt in der Rechtsprechung des EuGH zu den Grundfreiheiten}

Ein ähnliches Bild ergibt sich auch aus der Rechtsprechung des EuGH: Der grenzüberschreitende Bezug wird offenbar als notwendige Entscheidungsvoraussetzung angesehen, allerdings nicht genauer bestimmt.

Dies zeigt sich insbesondere an Urteilen, in denen der EuGH die Anwendung der Grundfreiheiten verneint, weil es in seinen Augen an einem grenzüberschreitenden Bezug fehlt. In der Vergangenheit handelte es sich dabei mehrfach um Urteile, in denen es um eine strafrechtliche Verurteilung eines Unionsbürgers ging. Der Gerichtshof nahm in diesen

3 Vgl. das Vorbringen der niederländischen Regierung in EuGH, Rs. 115/78, Knoors, Slg. 1979, 399, Rdnr. 24.

4 Von Bogdandy, in: Grabitz/Hilf/Nettesheim (Hrsg.), Das Recht der Europäischen Union, 50. EL 2013, Art. 18 AEUV, Rdnr. 1; Epiney, in: Calliess/Ruffert (Hrsg.), EUV/AEUV, 4. Aufl. 2011, Art. 18 AEUV, Rdnr. 1; Leopold/Semmelmann, Civis europaeus sum - Gewährleistungen und Grenzen der Freizügigkeit der Unionsbürger, ZEuS 2008, S. 291; Mestmäcker/Schweitzer, Europäisches Wettbewerbsrecht, 2. Aufl. 2004, § 2, Rdnr. 63; Hailbronner, Diskriminierungsverbot, Unionsbürgerschaft und gleicher Zugang zu Sozialleistungen, ZaöRV 2004, S. 603; Epiney, Umgekehrte Diskriminierungen, 1995, S. 91.

5 Kingreen, in: Ehlers (Hrsg.), Europäische Grundrechte und Grundfreiheiten, 3. Aufl. 2009, § 13, Rdnr. 2.

6 Vgl. ibid., Rdnr. 3. Auch von Bogdandy, (Fn. 4), Art. 18 AEUV, Rdnr. 1. 
Fällen an, dass diese Sachverhalte „,keinerlei über den rein innerstaatlichen Rahmen hinausweisende(n) Gesichtspunkte" aufweisen. ${ }^{7}$

Eine abstrakte Definition aber, unter die die zu entscheidenden Einzelfälle subsumiert werden könnten, hat sich in den letzten fünfzig Jahren nicht entwickelt. Dies lässt sich vor allem an den Grundfreiheiten gut verdeutlichen.

\section{Das Negativkriterium „kein rein interner Sachverhalt“"}

Bei den Grundfreiheiten geht der Gerichtshof in ständiger Rechtsprechung davon aus, dass diese ihre Wirkung nur dann entfalten können, wenn ein grenzüberschreitender Sachverhalt gegeben ist. Weisen die Merkmale eines zu beurteilenden Sachverhalts nicht über die Grenzen eines Mitgliedstaats hinaus, lehnt er eine Entscheidung ab: Die Grundfreiheiten seien ,nicht auf Betätigungen anwendbar, deren Elemente sämtlich nicht über die Grenzen eines Mitgliedstaats hinausweisen“" 8 Oder: Die Grundfreiheiten können nicht auf Sachverhalte angewendet werden, „die einen Mitgliedstaat rein intern betreffen, d.h., denen jeglicher Bezug zu irgendeinem Tatbestand fehlt, die das Gemeinschaftsrecht regelt. “9

Daran hält der EuGH auch trotz der Fortentwicklung der Grundfreiheiten zu Beschränkungsverboten fest. ${ }^{10}$ Generalanwalt Alber bringt das Spannungsverhältnis zwischen freiheitsrechtlichem Verständnis der Grundfreiheiten und damit einhergehenden Bedeutungsverlust des grenzüberschreitenden Sachverhalts auf den Punkt:

„Zwar ist auch für die Arbeitnehmerfreizügigkeit ein weiter Tatbestand eröffnet - vergleichbar der Dassonville-Formel - er ist jedoch bereits dadurch begrenzt, daß sich nur diejenigen auf die Freizügigkeit berufen können, die sie grenzüberschreitend in Anspruch nehmen." ${ }^{11}$

7 EuGH, Rs. 20/87, Gauchard, Slg. 1987, 4879; EuGH, Rs. 204/87, Bekaert, Slg. 1988, 2029; EuGH, verb. Rs. 54/88, 91/88 und C-14/89, Nino, Slg. 1990, I-3537; EuGH, Rs. 155/80, Oebel, Slg. 1981, 1993. Vgl. dazu Nachbaur, Niederlassungsfreiheit, 1999, S. 108.

8 EuGH, Rs. C-134/94, Esso, Slg. 1995, 4223, Rdnr. 13; EuGH, Rs. C-332/90, Steen I, Slg. 1992, I-341, Rdnr. 9; EuGH, Rs. C-29/94, Aubertin, Slg. 1995, I-301, Rdnr. 9; EuGH, Rs. C-132/93, Steen II, Slg. 1994, I-2715, Rdnr. 11; EuGH, verb. Rs. 35 und 36/82, Morson, Slg. 1982, 3723, Rdnr. 16; EuGH, Rs. C-206/91, Poirrez, Slg. 1992, I-6685, Rdnr. 11; ebenso EuGH, Rs. C-108/98, RI.SAN, Slg. 1999, I-5219; EuGH, Rs. C-97/98, Jaegerskioeld, Slg. 1999, I-7319. Umfangreiche Nachweise bei Epiney, in: Calliess/Ruffert, (Fn. 4), Art. 18 AEUV, Rdnr. 30.

9 EuGH, Rs. 175/78, Saunders, Slg. 1979, 1129, Rdnr. 11; EuGH, Rs. 180/83, Moser, Slg. 1984, 2539, Rdnr. 15; vgl. auch EuGH, Rs. C-212/06, Gouvernement de la Communauté français, Slg. 2008, I-1683, Rdnr. 32 ff.

10 Vgl. nur EuGH, Rs. C-275/92, Schindler, Slg. 1994, I-1039, Rdnr. 29. Zur Ausweitung der Grundfreiheiten auf Beschränkungsverbote vgl. statt vieler Behrens, Die Konvergenz der wirtschaftlichen Freiheiten im europäischen Gemeinschaftsrecht, EuR 1992, S. 148 ff.; Pache, in: Schulze/Zuleeg/Kadelbach (Hrsg.), Europarecht, Handbuch für die deutsche Rechtspraxis, 2. Aufl. 2010, § 10, Rdnr. 22; Streinz, Europarecht, 9. Aufl. 2012, Rdnr. 805. Die Position des EuGH in dieser Frage wird auch in der Literatur geteilt, vgl. nur Forsthoff, in: Grabitz/Hilf/ Nettesheim, (Fn. 4), Art. 45 AEUV, Rdnr. 54; Roth, in: Dauses, Handbuch EU-Wirtschaftsrecht, 33. EL 2013, E.I., Rdnr. 31. Frenz, Handbuch Europarecht, 2. Aufl. 2012, Bd. 1, Rdnr. 133 ff. Oppermann/Classen/Nettesheim, Europarecht, 5. Aufl. 2011, § 27, Rdnr. 42.

11 Schlussanträge GA Alber zu EuGH, Rs. C-176/96, Lehtonen, Slg. 2000, I-2681, Nr. 49. 
Offen bleibt dabei allerdings, was dies konkret heißt. Denn die Umschreibung des grenzüberschreitenden Sachverhalts mit einem nicht nur rein internen Sachverhalt ist im Kern nicht mehr als ein Zirkelschluss.

\section{Das Positivkriterium „Auswirkungen auf den innergemeinschaftlichen Handel“}

Wertet man verschiedene Urteile des EuGH zu den Grundfreiheiten aus, ${ }^{12}$ fällt auf, dass die berühmte „Dassonville“-Formel, mit der der Gerichtshof seinerseits die Warenverkehrsfreiheit (Art. 34 AEUV) vom Diskriminierungs- zum Beschränkungsverbot weitete, gleichzeitig auch das Verständnis des grenzüberschreitenden Sachverhalts fundamental verändert hat. ${ }^{13}$

Nach dieser Formel ist eine Maßnahme gleicher Wirkung wie eine mengenmäßige Beschränkung eine Handelsregelung, die ,geeignet ist, den innergemeinschaftlichen Handel unmittelbar oder mittelbar, tatsächlich oder potenziell zu behindern". ${ }^{14}$ Mit dem innergemeinschaftlichen Handel beinhaltet die Formel damit auch eine grenzüberschreitende Komponente: Entscheidend ist, welche Auswirkungen eine Maßnahme auf den innergemeinschaftlichen Handel hat.

Allerdings verändert sich dadurch bei genauerem Hinsehen auch der Blickwinkel: Referenzpunkt ist nicht mehr ein irgendwie gearteter Grenzübertritt, sondern der innergemeinschaftliche Handel. Die Beeinträchtigung oder der zugrundliegende Sachverhalt selbst muss nicht grenzüberschreitenden Charakter haben, es kommt nur darauf an, dass sich die Maßnahme oder die Handlung irgendwie auf den innergemeinschaftlichen Handel auswirken.

Der grenzüberschreitende Bezug erfährt dadurch eine Bedeutungsveränderung: Einer rein innerstaatlichen Maßnahme, die nur einen nationalen Markt tangiert, kann der grenzüberschreitende Bezug fehlen; Auswirkungen auf den innergemeinschaftlichen Handel kann sie gleichwohl haben. ${ }^{15}$ Es kommt nicht mehr auf den tatsächlichen Grenzübertritt eines Wirtschaftsfaktors oder die Staatsangehörigkeit einer Person an - sofern man zu Recht in diesen beiden Kriterien die Grundmerkmale eines grenzüberschreitenden Sachverhalts erblickt. ${ }^{16}$

Gerade das Merkmal der Staatsangehörigkeit scheint - wenn man die primärrechtlichen Aussagen zum Beispiel in Art. 18 AEUV ernstnimmt - bei den personenbezogenen Rechten (der Arbeitnehmerfreizügigkeit, Art. 45 AEUV, der Niederlassungsfreiheit, Art. 49 AEUV

12 So Lippert, Der grenzüberschreitende Sachverhalt im Unionsrecht, 2013, S. $44 \mathrm{ff}$.

13 Vgl. anschaulich EuGH, Rs. C-448/98, Guimont, Slg. 2000, I-10663, Rdnr. 14 ff.; EuGH, Rs. C-321/94, Pistre, Slg. 1997, I-2343, Rdnr. 41 ff.

14 EuGH, Rs. 8/74, Dassonville, Slg. 1974, 837, Rdnr. 5.

15 Vgl. Leible/Streinz, in: Grabitz/Hilf/Nettesheim, (Fn. 4), Art. 34 AEUV, Rdnr. 33. Vgl. Weyer, Freier Warenverkehr und nationale Regelungsgewalt in der Europäischen Union, 1997, S. 13 f. Auch Lach, Umgekehrte Diskriminierungen im Gemeinschaftsrecht, 2008, S. 86 ff.

16 Vgl. nur Kutzscher, (Fn. 1), S. 34 ff. Vgl. insbes. für das tatsächliche Überschreiten einer Grenze Schlussanträge GA Tesauro zu EuGH, verb. Rs. C-363/93, C-407/93, C-408/93, C-409/93, C-410/93 und C-411/93, Lancry, Slg. 1994, I-3957, Nr. 27; ähnlich Schlussanträge GA Jacobs zu EuGH, verb. Rs. C-321/94, C-322/94, C-323/94 und C-324/94, Pistre, Slg. 1997, I-2343, Nr. $33 \mathrm{ff}$. 
und dem unionsbürgerschaftlichen Freizügigkeitsrechts, Art. 20, 21 AEUV) - darauf angelegt zu sein, einen grenzüberschreitenden Bezug zu vermitteln, wenn sich der Staatsangehörige eines Mitgliedstaats gegenüber einem anderen Mitgliedstaat (also nicht gegenüber seinem eigenen Heimatstaat) auf seine Rechte beruft. ${ }^{17}$ Es müsste sich also um eine aus Sicht des Adressaten-Mitgliedstaats andere Staatsangehörigkeit handeln. ${ }^{18}$ Gleichwohl ist die Rechtsprechung des Gerichtshofs in diesem Punkt uneinheitlich und ohne klare Linie.

So hat er in der Rechtssache Werner den grenzüberschreitenden Sachverhalt verneint, obwohl Herr Werner in den Niederlanden wohnte, aber in Deutschland als selbständiger Zahnarzt arbeitete: Er könne sich gegenüber seinem eigenen Mitgliedstaat nicht auf die Grundfreiheiten berufen. ${ }^{19}$ In der Rechtssache Knoors hingegen hält der Gerichtshof fest, dass es für die personenbezogene Niederlassungsfreiheit nicht darauf ankomme, dass derjenige, der sich auf die Grundfreiheiten berufe, eine andere Staatsangehörigkeit habe, als der Mitgliedstaat, dem gegenüber er seine Rechte geltend macht. ${ }^{20}$ In der Rechtssache Scholz schließlich kommt der EuGH zu dem Ergebnis, dass jeder Unionsbürger ,unabhängig von seinem Wohnort und seiner Staatsangehörigkeit in den Anwendungsbereich“ der Grundfreiheiten fallen könne. ${ }^{21}$

Wenn der EuGH auf die Wirkungen einer Maßnahme abstellt, dann weitet das den Anwendungsbereich der Grundfreiheiten und damit auch die Kontrollbefugnis des EuGH. Denn dadurch, dass auch nur potentielle und mittelbare Auswirkungen erfasst sind, kann praktisch jede mitgliedstaatliche Maßnahme Auswirkungen auf den innergemeinschaftlichen Handel haben. ${ }^{22}$ Um diese Auswirkung beurteilen zu können, muss eine Bewertung anhand ökonomisch-empirischer Kriterien vorgenommen werden. ${ }^{23}$

\section{Die Weite des EuGH-Kriteriums}

Mit seinem Ansatz kann der EuGH auf flexible Art und Weise eine Vielzahl von Fallgestaltungen erfassen und den grenzüberschreitenden Bezug in jedem Einzelfall bejahen oder verneinen.

17 Vgl. dazu insbes. EuGH, Rs. C-112/91, Werner, Slg. 1993, I-429; EuGH, Rs. 175/78, Saunders, Slg. 1979, 1129.

18 Vgl. auch Frenz, (Fn. 10), Rdnrn. 134 f., 276.

19 EuGH, Rs. C-112/91, Werner, Slg. 1993, I-429, insbes. Rdnr. 16. Ähnlich in EuGH, Rs. 175/78, Saunders, Slg. 1979, 1129.

20 EuGH, Rs. 115/78, Knoors, Slg. 1979, 399, Rdnr. 24.

21 EuGH, Rs. C-419/92, Scholz, Slg. 1994, I-505, Rdnr. 9 ff.

22 Kingreen, in: von Bogdandy (Hrsg.), Europäisches Verfassungsrecht, 1. Aufl. 2003, S. 659. Weyer, (Fn. 15), S. 22 ff.

23 Mayer, Die Warenverkehrsfreiheit im Europarecht - eine Rekonstruktion, EuR 2003, S. 803: „Problematisch an einer solchen auf die Effekte einer Regelung abstellenden Betrachtung ist, dass hier Grenzen des Rechts erreicht werden, weil es letztlich um ökonomisch-empirische Daten geht.“ 


\section{a) Der Grenzübertritt in Vergangenheit und Zukunft}

Dies gilt zuerst für Sachverhalte, bei denen ein Grenzübertritt in der Vergangenheit lag bzw. erst noch in Zukunft vorliegen wird. Mit dem Ansatz des EuGH kommt es in zeitlicher Hinsicht nicht mehr darauf an, dass der grenzüberschreitende Bezug genau im Zeitpunkt der Berufung auf die jeweiligen Rechte vorlag.

\section{(1) Rückkehrerfälle}

Die Fallgestaltungen, bei denen der grenzüberschreitende Bezug in der Vergangenheit lag, kann man als Rückkehrerfälle bezeichnen: Hier hatte sich beispielsweise ein Staatsangehöriger in einen anderen Mitgliedstaat begeben, um dort eine Qualifikation oder ähnliches zu erwerben und kehrte dann in seinen Heimatstaat zurück. Dort wird ihm die erworbene Qualifikation nicht anerkannt, sodass er sich zur Abwendung dieser Beschränkung gegenüber seinem eigenen Mitgliedstaat auf die Grundfreiheiten beruft. ${ }^{24}$ Insofern ist diese Kategorie auch mit der oben bereits problematisierten Berufung eines Unionsbürgers auf die Grundfreiheiten gegenüber seinem eigenen Mitgliedstaat verbunden. Der eigene Staatsangehörige befinde sich zur Zeit der Berufung auf die grundfreiheitlichen Rechte gegenüber seinem eigenen Mitgliedstaat in einer einem ausländischen Staatsangehörigen vergleichbaren Lage; ${ }^{25}$ der in der Vergangenheit erfolgte Grenzübertritt wirkt für den EuGH praktisch fort. ${ }^{26}$

\section{(2) Wegzugsfälle}

Die Wegzugsfälle stellen in zeitlicher Hinsicht das Gegenstück zu den Rückkehrerfälle dar: ${ }^{27}$ In diesen Fallkonstellationen ist erst in Zukunft eine grenzüberschreitende Tätigkeit geplant. Dies ist zum Beispiel dann der Fall, wenn ein Unionsbürger von seinem eigenen Mitgliedstaat gehindert wird, in einem anderen Mitgliedstaat eine Arbeit aufzunehmen, sich dort niederzulassen oder eine Dienstleistung zu erbringen. ${ }^{28}$

In der Rechtssache Bosman beispielsweise berief sich der belgische Profifußballer Bosman gegenüber dem nationalen belgischen Fußballverband auf die Arbeitnehmerfreizügigkeit, weil ihn dessen Transferregelungen daran hindern würden, zu einem französischen Verein zu wechseln. Der EuGH bejaht den grenzüberschreitenden Bezug, weil Bosman in Zukunft in einen ausländischen Verein wechseln wollte: Die Staatsangehörigen der Mitgliedstaaten hätten das unmittelbar aus dem Vertrag abgeleitete Recht, ihr Herkunftsland

24 Vgl. oben EuGH, Rs. 115/78, Knoors, Slg. 1979, 399, Rdnr. 24; EuGH, Rs. C-419/92, Scholz, Slg. 1994, I-505; ebenso EuGH, Rs. 246/80, Broeckmeulen, Slg. 1981, 2311. Zum Begriff auch Haratsch/Koenig/Pechstein, Europarecht, 7. Aufl. 2010, Rdnr. 905. Lach, (Fn. 15), S. 70 f.

25 EuGH, Rs. 115/78, Knoors, Slg. 1979, 399, Rdnr. 24.

26 Forsthoff, (Fn. 10), Art. 45 AEUV, Rdnr. 59. Pache, (Fn. 10), § 10, Rdnr. 14. Vgl. Nachbaur, (Fn. 7), S. 79 ff.

27 Vgl. für die Differenzierung zwischen Zuzugs- und Wegzugsfälle im Rahmen der Arbeitnehmerfreizügigkeit auch Wienbracke, „Innerhalb der Union ist die Freizügigkeit der Arbeitnehmer gewährleistet"- eine aktuelle Bestandsaufnahme zu Art. 45 AEUV, EuR 2012, S. 496.

28 Ehlers, in: Ehlers, (Fn. 5), § 7, Rdnr. 24; Nachbaur, (Fn. 7), S. 112 f.; zum Begriff auch Haratsch/Koenig/Pechstein, (Fn. 24), Rdnr. 905. 
zu verlassen, um sich zur Ausübung einer wirtschaftlichen Tätigkeit in das Gebiet eines anderen Mitgliedstaats zu begeben. Werde dies verhindert, liege eine Beeinträchtigung dieser Freiheit vor, was insbesondere auch unabhängig von der Staatsangehörigkeit gelte. $^{29}$

Es reicht also die Absicht aus, in der Zukunft grenzüberschreitend tätig zu werden; der grenzüberschreitende Bezug wird in die Subjektivität des einzelnen Unionsbürgers verlagert, er ergibt sich nur mehr allein aus der „Fortbewegungsabsicht“ des Unionsbürgers. ${ }^{30}$

\section{b) Der hypothetische Grenzübertritt}

Die Fälle eines in der Zukunft liegenden grenzüberschreitenden Sachverhalts lassen sich auch unter die Kategorie des hypothetischen Grenzübertritts fassen.

In dieser Konstellation ist ein grenzüberschreitender Bezug auch in Zukunft nicht konkret erkennbar, sondern wird nur möglicherweise stattfinden. ${ }^{31}$ Auch dies reicht für den EuGH aus, korrespondiert doch diese Fallgestaltung mit den bloß potentiellen Beeinträchtigungen, die nach der „Dassonville“-Formel für eine Beeinträchtigung der Grundfreiheiten ausreichen sollen. ${ }^{32}$

Ein anschauliches Beispiel für diese Fallgruppe ist die Rechtsprechung zu den Goldenen Aktien. Sie umfasst zahlreiche Urteile, die sich allesamt mit der Frage befassen, ob bestimmte Regelungen, die dem Staat bei privatisierten Staatsbetrieben auch in Zukunft Einflussmöglichkeiten sichern sollen (z.B. Vorzugsaktien, Genehmigungsvorbehalte, Kontrollrechte, Mitwirkungsrechte bei gesellschaftsinternen Entscheidungen), mit Unionsrecht vereinbar sind. ${ }^{33}$ Der EuGH bejaht in allen Fällen - bis auf eine Ausnahme ${ }^{34}$ - einen Verstoß gegen die Kapitalverkehrsfreiheit. Diese sei bereits dann verletzt, wenn die zu beurteilende Maßnahme geeignet ist, den Kapital- oder Zahlungsverkehr zu verhindern oder ,illusorisch

29 EuGH, Rs. C-415/93, Bosman, Slg. 1995, 4921, Rdnr. 95 f.; vergleichbar EuGH, Rs. C-176/96, Lehtonen, Slg. 2000, I-2681 für die Basketballvereinigungen. Vgl. auch Weatherill, Annotation of the European Court's Judgment of 15 December 1995 - Case C-415/93, CMLRev. 33 (1996), S. 1019 ff. Ähnlich EuGH, Rs. C-190/98, Graf, Slg. 2000, I-493. Vgl. ausführlich Lippert, (Fn. 12), S. 53.

30 Frenz, Handbuch Europarecht, 1. Aufl. 2004, Bd. 1, Rdnr. 113 f.

31 Vgl. Lach, (Fn. 15), S.114. Vgl. Potacs, Effet utile als Auslegungsgrundsatz, EuR 2009, S. 481 f., der insoweit von einer „,bloß juristisch-formale(n) Inanspruchnahme der Grundfreiheiten“" spricht, bei der der EuGH eine Betätigung schützt, die „faktisch nicht ausgeübt wird“.

32 Vgl. Gebauer, Die Grundfreiheiten des EG-Vertrags als Gemeinschaftsgrundrechte, 2004, S. 91; Papadileris, (Fn. 1), S. 124 f.; vgl. Lach, (Fn. 15), S. 181.

33 Vgl. dazu Lippert, Der EuGH und die Goldenen Aktien, Jura 2009, S. 342; auch Ruge, Goldene Aktien und EG-Recht, EuZW 2002, S. 421; Roth, in: Roth/Hilpold, Der EuGH und die Souveränität der Mitgliedstaaten, 2008, S. 430 f.; Spindler, Deutsches Gesellschaftsrechtsrecht in der Zange zwischen Inspire Art und Golden Shares?, RIW 2003, S. 850.

34 EuGH, Rs. C-503/99, Kommission/Belgien, Slg. 2002, I-4809. Zusammenfassend, Lippert, (Fn. 33), S. 345. Die aktuellste Entscheidung des EuGH zum deutschen VW-Gesetz (Urt. v. 22.10.2013, Rs. C-95/12) kann im vorliegenden Zusammenhang nicht als Ausnahme gewertet werden, da das Urteil in einem Verfahren nach Art. 260 Abs. 2 AEUV erging, also nur festzustellen war, ob die Bundesrepublik Deutschland die entsprechenden, aus dem ursprünglichen Urteil des EuGH (Urt. v. 23.10.2007, Rs. C-112/05) folgenden Maßnahmen ergriffen hat. 
zu machen“35 oder „Anleger aus anderen Mitgliedstaaten von [...] Investitionen abzuhalten“ ${ }^{36}$ oder „,den Erwerb von Aktien der betreffenden Unternehmen zu verhindern oder zu beschränken oder aber Investoren anderer Mitgliedstaaten davon abzuschrecken, in das Kapital dieser Unternehmen zu investieren“. ${ }^{3738}$ In allen Fällen war jedoch nicht erkennbar, dass ein (ausländischer) Investor konkret in ein Unternehmen, für das ein staatliches Vorzugsrecht bestand, investieren wollte. Der grenzüberschreitende Sachverhalt ergab sich für den Gerichtshof allein daraus, dass möglicherweise, also hypothetisch, ein grenzüberschreitendes Investment stattfinden könnte.

\section{c) Der wirtschaftliche Vergleichsmaßstab}

Geht man einen Schritt weiter und lässt auch den hypothetischen Grenzübertritt hinter sich, zeichnet sich in der Rechtsprechung des EuGH eine weitere Kategorie ab, die noch weniger mit einem grenzüberschreitenden Bezug verbunden ist: Sie soll hier als wirtschaftlicher Vergleichsmaßstab bezeichnet werden, ${ }^{39}$ da hier nur noch die wirtschaftlichen Auswirkungen von beschränkenden Maßnahmen in den Blick genommen werden.

Ein anschauliches Beispiel für diese Konstellation ist die Rechtssache Keck. Anhand dieser Entscheidung hat der EuGH die Unterscheidung von Verkaufs- und Produktmodalitäten eingeführt und letztere aus dem Anwendungsbereich der Grundfreiheiten herausgenommen. In tatsächlicher Hinsicht ging es in der Rechtssache Keck darum, dass im Elsass ansässige, französische Supermärkte in dem Verbot, Waren unter Einkaufspreis zu verkaufen, einen Verstoß gegen Art. 34 AEUV erblickten. Der Gerichtshof prüft die Zulässigkeit dieser Regelung „unter dem Blickwinkel des freien Warenverkehrs“ “40 Er muss also vom Vorliegen eines grenzüberschreitenden Sachverhalts ausgehen, selbst wenn er die Verkaufsmodalitäten aus dem Tatbestand der Warenverkehrsfreiheit herausnimmt: Die Tatbestandsrestriktion setzt zunächst das Vorliegen der übrigen Merkmale des Tatbestands voraus. Dies bedeutet, dass das grenzüberschreitende Element nur darin bestehen kann, dass - wie die Parteien dies vorbrachten - durch das beschriebene Verbot der Warenumsatz und damit die Ausweitung des grenzüberschreitenden Handels behindert würde, also die Parteien tatsächlich Waren aus anderen Mitgliedstaaten beziehen; dies war bei den in der Rechtssache Keck betroffenen Supermärkten an der deutsch-französischen Grenze der Fall.

35 EuGH, Rs. C-483/99, Kommission/Frankreich, Slg. 2002, I-4781, Rdnr. 41.

36 EuGH, Rs. C-463/00, Kommission/Spanien, Slg. 2003, I-4581, Rdnr. 61.

37 EuGH, Rs. C-282/04, Kommission/Niederlande, Slg. 2006, I-9141, Rdnr. 20.

38 Diese Attraktivitätsminderung war auch das wesentliche Argument, mit dem der EuGH die deutschen Vorzugsrechte für den Autohersteller Volkswagen im so genannten VW-Gesetz verwarf, EuGH, Rs. C-112/05, VW-Gesetz, Slg. 2007, I-8995, Rdnr. 52. Dazu Lippert, (Fn. 33), S. 346; auch Reich, Kurzbesprechung der Schlussanträge von Generalanwalt Dámaso Ruiz-Colomer v. 13. 2. 2007 in der Rs. C-112/05 - Kommission/Bundesrepublik Deutschland betreffend das VW-Gesetz (VWG), EuZW 2007, S. 132; Roth, (Fn. 33), S. 435 f.

39 Ausführlich Lippert, (Fn. 12), S. $57 \mathrm{ff}$.

40 EuGH, verb. Rs. C-267/91 und C-268/91, Keck und Mithouard, Slg. 1993, I-6079, Rdnr. 10. Ähnlich EuGH, Rs. C-254/98, TK-Heimdienst Sass GmbH, Slg. 2000, I-151, Leitsatz. Vgl. dazu ausführlich Lippert, (Fn. 12), S. 57. 
Es ist aber fraglich, ob dies genügen kann; denn ein Kiosk, der nur ein kleines Sortiment von einheimischen Waren anbietet, könnte sich dann nicht auf Art. 34, 36 AEUV berufen. ${ }^{41}$

Ein weiteres anschauliches Beispiel bietet die Entscheidung des EuGH zum deutschen Einwegsystem für Mineralwasserflaschen: Deutschland schrieb ab 2003 die Erhebung eines Pfandes auf Einwegverpackungen für Mineralwasser, Bier und kohlensäurehaltige Erfrischungsgetränke vor, weil der Mehrweganteil bei Getränkeverpackungen ab 1997 unter eine gesetzlich vorgeschriebene Quote fiel. Die Kommission sah darin eine Beeinträchtigung der Warenverkehrsfreiheit, weil die Hersteller von Mineralwasser, die an der Quelle abgefüllt werden, die leere Verpackung zur Wiederverwertung an den Abfüllort zurückbefördern müssten. Der EuGH folgt im Wesentlichen der Argumentation der Kommission. Die Schlechterstellung beruhte nicht auf der grenzüberschreitenden Tätigkeit der Unternehmen; das Problem der Rückbeförderung über längere Strecken trifft den inländischen Wasserabfüller, der seine Flaschen möglicherweise durch ganz Deutschland transportieren muss, härter, als den grenznahen Anbieter. Ausländische Anbieter sind nach Auffassung des EuGH allein deshalb stärker betroffen, weil nach einer Studie aus dem Jahre 2001 ausländische Hersteller von Mineralwasser in weit größerem Umfang als deutsche Hersteller Einwegverpackungen nutzten. ${ }^{42}$ Der Gerichtshof verwendet in diesem Fall also statistische Quellen zum Nachweis des grenzüberschreitenden Sachverhalts; allein die unterschiedliche Quantität der Verwendung bestimmter Verpackungen reicht aus, um einen grenzüberschreitenden Bezug zu begründen. Sein Referenzkriterium ist damit kein qualitatives, sondern ein quantitatives geworden. ${ }^{43}$

\section{Das Ziel: Unionsweite Mobilität - Das Mittel: Der ökonomisch relevante Sachverhalt}

\section{a) Das Ziel des EuGH: Unionsweite Mobilität}

Welches Ziel verfolgt der EuGH mit seinem Ansatz? Die Antwort liegt auf der Hand: Es geht um die Schaffung und Sicherstellung einer umfassenden Mobilität auf dem gesamten Binnenmarkt. ${ }^{44}$ Wenn sich die Rechtsprechung des Gerichtshofs hinsichtlich der Annahme oder Ablehnung des grenzüberschreitenden Sachverhalts in den konkreten Fallgestaltungen kaum rechtssicher vorhersagen lässt - das Ziel einer umfassenden Mobilität auf dem Binnenmarkt bleibt immer gleich.

An den Grundfreiten ließ sich dies anschaulich zeigen: Der EuGH musste den Blick von der spezifisch grenzüberschreitenden Konstellation lösen und auf den innergemeinschaft-

41 Vgl. Gebauer, (Fn. 32), S. 91 f.

42 EuGH, Rs. C-463/01, Deutsche Pfandflaschen, Slg. 2004, I-11705, Rdnr. 57 ff.

43 Vgl. Kingreen, (Fn. 22), S. 668; vgl. dazu auch Mayer, (Fn. 23), S. 803, der eine Verbindung zwischen Auslegung der Grundfreiheiten als Beschränkungsverbote und „ökonomisch-empirischer" Datengrundlage herstellt. Auch Lach, (Fn. 15), S. 41. Aus der Rspr. vgl. auch EuGH, Rs. 33/88, Allué I, Slg. 1989, 1591, Rdnr. $11 \mathrm{f}$.

44 Vgl. Nettesheim, Die europarechtlichen Grundrechte auf wirtschaftliche Mobilität (Art. 48, 52 EGV), NVwZ 1996, S. 342, der hinsichtlich der Grundfreiheiten sogar von „Grundrechten auf wirtschaftliche Mobilität" spricht. Vgl. auch Kingreen, in: von Bogdandy/Bast, Europäisches Verfassungsrecht: Theoretische und dogmatische Grundzüge, 2. Aufl. 2009, S. 722. 
lichen Handel bzw. den Binnenmarkt in Gänze richten. Es dürfen nicht nur spezifisch grenzüberschreitende Behinderungen abgebaut werden, sondern alle Beeinträchtigungen, die allgemein die Zirkulation von Wirtschaftsgütern behindern. Dies führt dazu, dass auch mitgliedstaatliche Regulierungen ,hinter der Grenze“, d.h. Regelungen, die nicht spezifisch an grenzüberschreitende Sachverhalte anknüpfen, sondern für alle Wirtschaftsteilnehmer gleichermaßen gelten, in den Fokus der Rechtsprechung geraten. ${ }^{45}$

Nur so lässt sich die Annahme eines grenzüberschreitenden Sachverhalts in den obigen Kategorien erklären. Bei den Rückkehrerfällen beispielsweise kann umfassende Mobilität nur hergestellt werden, wenn eine im Ausland erworbene berufliche Qualifikation auch nach Rückkehr in den Heimatstaat anerkannt wird. Die wirtschaftliche Mobilität auf dem gesamten Binnenmarkt kann nur durch eine vollständige Marktintegration hergestellt werden. Erst dann ist eine unbeschränkte Bewegung aller Marktteilnehmer auf dem Binnenmarkt möglich.

Damit treten an wirtschaftlichen Kriterien orientierte, quantitative Merkmale in den Vordergrund: Ein grenzüberschreitender Bezug wird schon dann bejaht, wenn nachteilige Auswirkungen auf den Wirtschaftsverkehr in Frage stehen - unabhängig von der Frage, ob die zugrundliegende Wirtschaftstätigkeit grenzüberschreitenden Charakter hat oder nicht. Nicht mehr die rechtlichen Kriterien des Übertritts einer Grenze zwischen zwei Mitgliedstaaten im staatsrechtlichen Sinne, sondern die Veränderung einer quantitativen Kennziffer reicht aus. Grenzüberschreitend ist nunmehr ein Sachverhalt, der ökonomisch relevant ist. $^{46}$

\section{b) Die Vermutungsregel}

Vor diesem Hintergrund erscheint es aus Sicht des EuGH konsequent, grundsätzlich eher zu Gunsten eines grenzüberschreitenden Bezugs zu entscheiden. Das Vorliegen eines grenzüberschreitenden Sachverhalts wird in der Rechtsprechung zum Regelfall und nicht zur begründungsbedürftigen Ausnahme.

Dies erklärt dann auch, warum der Ansatzpunkt des EuGH ein negativer ist: Ein grenzüberschreitender Bezug ist gegeben, wenn kein rein interner Sachverhalt vorliegt. ${ }^{47}$ Der grenzüberschreitende Bezug erschließt sich damit immer nur über die Verneinung eines rein internen Sachverhalts. Immer dann, wenn ein rein interner Sachverhalt nicht vorliegt, wird ohne weiteres ein grenzüberschreitender Bezug bejaht. Damit wird der negative Definitionsansatz zugleich zu einer Vermutungsregel: Es wird vermutet, dass bei fehlendem rein internen Sachverhalt ein grenzüberschreitender Bezug gegeben ist. ${ }^{48}$

45 Oppermann/Classen/Nettesheim, (Fn. 10), § 28, Rdnr. 34.

46 Begriff von Calliess, Der Unionsbürger: Status, Dogmatik und Dynamik, EuR-Beiheft 1/2007, S. 19 unter Verweis u.a. auf Ipsen, Europäisches Gemeinschaftsrecht, 1972, S. 9/136. Ähnlich Grabitz, Europäisches Bürgerrecht zwischen Marktbürgerschaft und Staatsbürgerschaft, 1970, S. 69. Vgl. auch Weyer, (Fn. 15), S. 14 f., 17 f.

47 Vgl. dazu Schlussanträge GA Maduro zu EuGH, Rs. C-72/03, Carbonati, Slg. 2004, I-8027, Nr. 4; Schlussanträge GA Jacobs zu EuGH, verb. Rs. C-321/94, C-322/94, C-323/94 und C-324/94, Pistre, Slg. 1997, I-2343, Nr. 36 f. Dazu auch Plötscher, Der Begriff der Diskriminierung im Europäischen Gemeinschaftsrecht, 2003, S. 302.

48 Büchele, in: Roth/Hilpold, (Fn. 33), S. 351. 
Eine solche Herangehensweise bürgt zwei Gefahren. Zum einen droht die Aussage konturenlos zu werden, da sie sich auf eine reine Negation beschränkt, nicht aber allgemeingültige Kriterien entwickelt. ${ }^{49}$ Zum anderen dreht sie die Argumentationslast um, indem negativ dargelegt werden muss, dass kein rein interner Sachverhalt vorliegt, statt positiv den grenzüberschreitenden Bezug zu bejahen. ${ }^{50}$ Die Verneinung eines grenzüberschreitenden Bezugs ist wesentlich einfacher. Die positive Darstellung bestimmter Voraussetzungen setzt eine erhöhte Argumentationsleistung voraus, der der Gerichtshof mit seiner Konstruktion ausweicht. ${ }^{51}$

Aber selbst diese positive Annahme eines grenzüberschreitenden Bezugs erleichtert das Kriterium des EuGH: Durch die Orientierung an der „Dassonville“-Formel reicht allein die Möglichkeit einer grenzüberschreitenden Auswirkung aus. Der grenzüberschreitende Sachverhalt ist zu einem Gefährdungstatbestand geworden. ${ }^{52}$

Diese Entwicklung erscheint im Wesentlichen aus zwei Gründen problematisch: Zum einen erweist sie sich als rechtsunsicher. Dies liegt nicht zuletzt an der Gleichsetzung von grenzüberschreitendem und ökonomisch relevantem Sachverhalt. Denn die Bezugnahme auf wirtschaftliche Sachverhalte, die nicht nur als Entscheidungshilfe im Rahmen rechtlicher Entscheidungen dienen, sondern diese ersetzen, ist immer mit einem hohen Grad an Rechtsunsicherheit belastet. ${ }^{53}$ Wirtschaftliche Sachverhalte lassen sich nicht anhand von vorgegeben Kriterien als richtig oder falsch beurteilen; sie sind ungeeignet, eine Entscheidung über zulässiges, gebotenes oder unzulässiges, verbotenes Verhalten zu fällen. ${ }^{54}$ Auch lässt sich ein Bezug zum Binnenmarkt nahezu immer herstellen. ${ }^{55}$ Hinzu kommt der Begriff

49 So auch Seyr/Rümke, Das grenzüberschreitende Element in der Rechtsprechung des EuGH zur Unionsbürgerschaft, EuR 2005, S. 674. Zum Argumentationsmuster der Negation in Urteilen des EuGH auch Heermann, Artikel 30 EGV im Lichte der „Keck“-Rechtsprechung: Anerkennung sonstiger Verkaufsmodalitäten und Einführung eines einheitlichen Rechtfertigungstatbestands?, GRURInt 1999, S. 587.

50 Die Beweis- und die Darlegungslast werden im Unionsrecht nicht so strikt getrennt wie im deutschen Prozessrecht; eine entsprechende Regelung gilt immer für die Substantiierung des Vortrags und zugleich auch für den vollen Nachweis, vgl. Schorkopf, Beweislast im Recht des freien Warenverkehrs - Die erneuerte Cassis-Formel als Schnittmenge von Binnenmarktrecht und GATT, EuR 2009, S. 647, Fn. 10.

51 Vgl. diesbezüglich z.B. die Argumentation in EuGH, Rs. C-200/02, Zhu und Chen, Slg. 2004, I-9925, Rdnr. 19: Die Argumentation des EuGH erschöpft sich darin, dass die Situation eines Unionsbürgers, der nie von seinem Freizügigkeitsrecht Gebrauch gemacht habe, nicht mit einer rein innerstaatlichen Situation gleichgestellt werden könne. Vgl. ähnlich Hailbronner, Die Unionsbürgerschaft und das Ende rationaler Jurisprudenz durch den EuGH?, NJW 2004, S. 2186. Vgl. auch Borchardt, Die rechtlichen Grundlagen der Europäischen Union, 5. Aufl. 2012, Rdnr. 915. Ähnlich auch Roth, (Fn. 33), S. 455 f., der aber mit dem Subsidiaritätsprinzip argumentiert.

52 Frenz, Handbuch Europarecht, Bd. 2, Europäisches Kartellrecht, 2006, Rdnr. 1402.

53 Vgl. Drexl, in: von Bogdandy/Bast, (Fn. 44), S. 933 ff. zum more economic approach in der Wettbewerbspolitik.

54 Vgl. Behrens, in: Behrens/Braun/Nowak, Europäisches Wettbewerbsrecht nach der Reform, 2006, S. 13.

55 Vgl. ähnlich bzgl. dem Ansatz der Kommission, ausschließliche Gemeinschaftskompetenzen bei einer Nähe des Sachgegenstandes zum Binnenmarkt anzunehmen Calliess, Subsidiaritätsprinzip und Solidaritätsprinzip in der Europäischen Union, 2. Aufl. 1999, S. 83 f. 
der Auswirkung, der eine Prognoseentscheidung verlangt. ${ }^{56}$ Eine solche Prognose ist aber notwendigerweise mit Unsicherheit und damit im Hinblick auf die rechtliche Beurteilung eines Sachverhalts auch mit großer Rechtsunsicherheit belastet. ${ }^{57}$

Zum anderen ist die Europäische Union von ihrer historischen Anlage und von ihrer rechtlich-systematischen Ausrichtung her als besondere Form der Zusammenarbeit der Mitgliedstaaten angelegt, die bestimmte Problembereiche einer gemeinsamen Lösung zuführen wollten. Bei diesen Problemen handelt es sich grundsätzlich um Fragestellungen, die besser auf überstaatlicher Ebene gelöst werden können, weil ein einzelner Staat dies nicht ebenso effektiv schaffen würde. Dies bedeutet aber, dass die grenzüberschreitende Tätigkeit der Union immer rechtfertigungsbedürftig ist und nicht - anders herum - die originäre, einzelstaatliche Verantwortung der Mitgliedstaaten. ${ }^{58}$

\section{Der grenzüberschreitende Sachverhalt in der Rechtsprechung des EuGH zum Wettbewerbsrecht}

Die Wettbewerbsregeln der Union enthalten - anders als die Grundfreiheiten - einen deutlicheren Anknüpfungspunkt für den grenzüberschreitende Sachverhalt im Tatbestand: Die Art. 101, 102, 107 AEUV verbieten bestimmte Verhaltensweisen, die den „Handel zwischen den Mitgliedstaaten" beeinträchtigen.

Diese Zwischenstaatlichkeitsklausel wird vom EuGH ebenfalls nach dem Vorbild der „Dassonville“-Formel ausgelegt, sodass eine wettbewerbsbeschränkende Maßnahme schon dann unter das Kartellrecht fällt, wenn sie

„unmittelbar oder mittelbar, tatsächlich oder der Möglichkeit nach den Warenverkehr zwischen Mitgliedstaaten beeinflussen kann." ${ }^{\text {59 }}$

56 Vgl. Drexl, (Fn. 44), S. 935. Zur Bedeutung der Prognose im Rahmen der ökonomischen Analyse und Auslegung des Rechts vgl. nur Hoeren/Stallberg, Grundzüge der Rechtsphilosophie, 2001, Rdnr. 268 ff.; Kirchner, in: Riesenhuber, Europäische Methodenlehre - Grundfragen der Methode des Europäischen Privatrechts, 2000, S. 37 f.

57 Ausdrücklich zur Wertungsabhängigkeit von Prognoseentscheidungen Koch, Der Grundsatz der Verhältnismäßigkeit in der Rechtsprechung des Gerichtshofs der Europäischen Gemeinschaften, 2003, S. 207. Vgl. auch Jarass, Die Kompetenzverteilung zwischen der Europäischen Gemeinschaft und den Mitgliedstaaten, AöR 1996, S. 180. Nothdurft, Ökonomie vor Gericht, Richterliche Überprüfung wirtschaftlicher Fragen im deutschen und europäischen Kartellverwaltungsprozess, Festschrift Hirsch, 2008, S. 291.

58 Dazu ausführlich Lippert, (Fn. 12), S. 279.

59 Für Art. 101 AEUV: EuGH, Rs. 56/65, Maschinenbau Ulm, Slg. 1966, 281, 303; EuGH, verb. Rs. 56 und 58/64, Consten Grundig, Slg. 1966, 321, 389. Für Art. 102 AEUV: EuGH, Rs. 22/78, Hugin, Slg. 1979, 1869, Rdnr. 17; EuGH, Rs. 85/76, Hoffmann-La Roche, Slg. 1979, 461, Rdnr. 125; EuG, Rs. T-24/93, Compagnie maritime belge transports, Slg. 1996, II-1201, Rdnr. 201; EuG, Rs. T-70/89, BBC, Slg. 1991, II-535, Rdnr. 64. Jung, in: Grabitz/Hilf/Nettesheim, (Fn. 4), Art. 102 AEUV, Rdnr. 361. Für Art. 107 AEUV: EuGH, Rs. 730/79, Philip Morris, Slg. 1980, 2671, Rdnr. 12; EuGH, Rs. C-126/01, Gemo, Slg. 2003, I-13769, Rdnr. 41; EuGH, Rs. C-222/04, Cassa di Risparmio di Firenze SpA, Slg. 2006, I-289, Rdnr. 141; EuG, Rs. T-214/95, Vlaams Gewest, Slg. 1998, II-717, Rdnr. 50; Mestmäcker/Schweitzer, (Fn. 4), § 42, Rdnr. 10. 
Zentral ist auch hier, dass die bloße Geeignetheit zur grenzüberschreitenden Auswirkung ausreicht und dass auch hier die Auswirkungen einer Maßnahme im Vordergrund stehen. ${ }^{60}$ Der Bezugspunkt dieser Auswirkungen ist der gesamte Binnenmarkt. ${ }^{61}$

Mittelbar ist eine Beeinträchtigung beispielsweise dann, wenn sich die Verhaltensweise nicht direkt auf den Handel mit dem Produkt selbst bezieht. ${ }^{62}$ Dies kann bei Zwischenerzeugnissen der Fall sein, wenn nur das Endprodukt Gegenstand des innergemeinschaftlichen Handels ist; ${ }^{63}$ ebenso, wenn sich Ausschließlichkeitsverträge oder Wettbewerbsverbote nur auf den innerstaatlichen Handel beziehen, dadurch aber mittelbar auf den zwischenstaatlichen Handel Auswirkungen haben, indem sie die Importe aus anderen Mitgliedstaaten tangieren. ${ }^{64}$ Damit werden über das Merkmal der mittelbaren Beeinträchtigung auch rein innerstaatliche Verhaltensweisen erfasst, sofern sie möglicherweise den Markt für ausländische Konkurrenten abschotten. ${ }^{65}$

Eine potentielle Beeinträchtigung liegt dann vor, wenn der Wettbewerb aus strukturellen Gründen behindert wird. Es reicht aus, wenn Auswirkungen auf den zwischenstaatlichen Handel mit hinreichender Wahrscheinlichkeit in Zukunft entstehen könnten, ${ }^{66}$ beispielsweise wenn durch die Änderungen der Marktbedingungen später einmal ein sehr stark innerstaatlich verwurzeltes Produkt (landessprachliches Kulturprodukt) in größerem Umfang ins Ausland exportiert wird. ${ }^{67}$

Gerade im Wettbewerbsrecht zeigt sich die an quantitativen Merkmalen orientierte Auslegung der Zwischenstaatlichkeitsklausel. Nicht umsonst wird sie in der Regel zusammen mit dem Tatbestandsmerkmal der Wettbewerbsbeeinträchtigung erörtert. Letzteres ist naturgemäß stark wirtschaftlich konnotiert; die dort verwendeten Kriterien werden auch auf die Zwischenstaatlichkeit übertragen. Dies zeigt sich insbesondere am so genannten more economic approach („ökonomischerer Ansatz“). ${ }^{68}$ Dieser Ansatz erfasst alle Tatbestände des Wettbewerbsrechts und zielt auf eine stärkere Einbeziehung wirtschaftswissenschaft-

60 Vgl. nur Mitteilung der Kommission, Leitlinien über den Begriff der Beeinträchtigung des zwischenstaatlichen Handels in den Artikeln 81 und 82 des Vertrags, ABl. C 101 v. 27.4.2004, S. 81 (im Folgenden: Leitlinien Zwischenstaatlichkeit), Rdnr. 21.

61 EuGH, Rs. 56/65, Maschinenbau Ulm, Slg. 1966, 281, 303. Mestmäcker/Schweitzer, (Fn. 4), § 4, Rdnr. 8. Zimmer, in: Immenga/Mestmäcker, Wettbewerbsrecht, Bd. 1, 5. Aufl. 2012, Art. 101 Abs. 1 AEUV, Rdnr. 199.

62 Vgl. Leitlinien Zwischenstaatlichkeit, (Fn. 60), Rdnr. 38 ff.

63 EuGH, Rs. 123/83, BNIC, Slg. 1985, 391, Rdnr. 29; EuGH, verb. Rs. C-89/85, C-104/85, C-114/85, C-116/85, C-117/85 und C-125/85 bis C-129/85, Ahlström, Slg. 1993, I-1307, Rdnr. 142.

64 EuGH, Rs. 126/80, Salonia, Slg. 1981, 1563, Rdnr. $11 \mathrm{ff}$.

65 Vgl. Zimmer, (Fn. 61) Art. 101 Abs. 1 AEUV, Rdnr. 217; Mestmäcker/Schweitzer, (Fn. 4), § 4, Rdnr. 18.

66 Leitlinien Zwischenstaatlichkeit, (Fn. 60), Rdnr. 41; Kirchhoff, in: Münchener Kommentar zum Europäischen und Deutschen Wettbewerbsrecht (Kartellrecht), Bd. 1, Europäisches Wettbewerbsrecht, 2007, Art. 81 EG, Rdnr. 584 ff.

67 EuGH, Rs. 19/77, Miller, Slg. 1978, 131, Rdnr. 14; vgl. auch EuGH, Rs. 107/82, AEG, Slg. 1983, 3151, Rdnr. 60.

68 Vgl. Christiansen, Die „Ökonomisierung“ der EU-Fusionskontrolle: Mehr Kosten als Nutzen?, WuW 2005, S. 292. 
licher Bewertungsmaßstäbe in die wettbewerbsrechtliche Kontrollpraxis ab. ${ }^{69}$ Es geht dabei um eine Bewertung der Effekte wettbewerblich relevanten Handelns anhand empirischer Daten. Dies erlaubt eine quantitative Abschätzung der Auswirkungen. ${ }^{70}$ Deswegen wird der more economic approach auch als auswirkungsbezogener Ansatz (effects-based approach) bezeichnet. ${ }^{71}$

Darüber hinaus macht auch das Merkmal der Spürbarkeit, mit der die Weite der zwischenstaatlichen Handelsbeeinträchtigung eingeschränkt werden soll, ${ }^{72}$ die quantitative Ausrichtung deutlich. Dieses Kriterium fragt nach der Schwere einer Wettbewerbsbeeinträchtigung. ${ }^{73}$ Die Kommission greift zur Bemessung auf quantitative Kriterien zurück: Eine Beeinträchtigung des zwischenstaatlichen Handels ist zu verneinen, wenn eine Marktanteilsschwelle von $5 \%$ nicht überschritten wird; sie soll vorliegen, wenn die zu beurteilende Vereinbarung einen Warenumsatz von mehr als 40 Mio. Euro erfasst oder die Marktanteilsschwelle von $5 \%$ überschreitet. ${ }^{74}$

\section{Quantitative Kriterien und Subsidiarität am Beispiel der Fusionskontrolle}

Der grenzüberschreitende Sachverhalt wird also offenbar - das zeigt sich an der Rechtsprechung des EuGH zu den Grundfreiheiten, aber auch im Wettbewerbsrecht - quantitativ, nach ökonomischen Kriterien bestimmt. Entscheidend ist weniger, ob ein Sachverhalt tatsächlich zwei oder mehr Mitgliedstaaten oder die Staatsangehörigen anderer Mitgliedstaaten betrifft, sondern vielmehr, ob ein ökonomisch relevanter Sachverhalt gegeben ist, der sich mit Zahlenwerten, Verhältnismäßigkeiten und Auswirkungen beschreiben lässt.

69 Vgl. nun auch die Erstreckung des more economic approach auf Art. 102 AEUV durch die Mitteilung der Kommission, Erläuterungen zu den Prioritäten der Kommission bei der Anwendung von Artikel 82 des EG-Vertrages auf Fälle von Behinderungsmissbrauch durch marktbeherrschende Unternehmen, ABl. C 45 v. 24.2.2009, S. 7; dazu Möschel, Der Missbrauch marktbeherrschender Stellungen nach Art. 82 EG-Vertrag und der „More Economic Approach“, JZ 2009, S. 1040; Frenz/Ehlenz, Defizitäre gerichtliche Wettbewerbskontrolle durch More-economicApproach und Vermutungen?, EuR 2010, S. 491.

70 Christiansen, (Fn. 68), S. 285; Schmidtchen, Der „more economic approach“ in der Wettbewerbspolitik, WuW 2006, S. 6; Frenz/Ehlenz, (Fn. 69), S. 491.

71 Drexl, (Fn. 44), S. 920; Möschel, (Fn. 69), S. 1040. Zu den Problemen im Rahmen von wettbewerbsrechtlichen Abwägungsentscheidungen vgl. Frenz/Ehlenz, (Fn. 69), S. 490.

72 Vgl. für Art. 101 AEUV nur EuGH, Rs. 5/69, Völk, Slg. 1969, 295, Rdnr. 7; EuGH, Rs. 22/71, Béguelin, Slg. 1971, 949, Rdnr. 16/18; EuGH, Rs. C-215/96, Bagnasco, Slg. 1999, I-135, Rdnr. 34; Frenz, (Fn. 52), Rdnr. 493. Für Art. 102 AEUV ist die Rspr. uneinheitlich, vgl. nur EuGH, verb. Rs. C-241/91 P und C-242/91 P, Magill, Slg. 1995, I-743, Rdnr. 69; EuG, Rs. T-24/93, Compagnie maritime belge transports, Slg. 1996, II-1201, Rdnr. 201; EuG, Rs. T-69/89, RTE, Slg. 1991, II-485, Rdnr. 77; EuG, Rs. T-70/89, BBC, Slg. 1991, II-535, Rdnr. 65; EuG, Rs. T-228/97, Irish Sugar, Slg. 1999, II-2969, Rdnr. 170 einerseits und EuGH, Rs. C-41/90, Höfner, Slg. 1991, I-1979, Rdnr. 32 andererseits. Aus der Literatur vgl. dazu nur Weiß, in: Calliess/Ruffert, (Fn. 4), Art. 102 AEUV, Rdnr. 73; Jung, (Fn. 59), Art. 102 AEUV, Rdnr. 369. Für Art. 107 AEUV und zur Diskussion allgemein vgl. Lippert, (Fn. 12), S. 131.

73 Vgl. Bunte, in: Langen/Bunte, Kommentar zum deutschen und europäischen Kartellrecht, Bd. 2, Europäisches Kartellrecht, 11. Aufl. 2010, Art. 81 EG, Rdnr. 151. Leitlinien Zwischenstaatlichkeit, (Fn. 60), Rdnr. 44 f.; dazu auch Bechtold/Bosch/Brinker/Hirsbrunner, EG-Kartellrecht, 2. Aufl. 2009, Art. 81 EG, Rdnr. 110.

74 Leitlinien Zwischenstaatlichkeit, (Fn. 60), Rdnr. 46 ff. 


\section{Die Aufgreifschwelle in der Fusionskontrolle als quantitatives Kriterium}

Bestätigt wird diese Beobachtung auch durch die Regelungen zur europäischen Fusionskontrolle. Die EG-Fusionskontrollverordnung ${ }^{75}$ ist nach ihrem Art. 1 nur anwendbar auf Zusammenschlüsse von Unternehmen mit gemeinschaftsweiter Bedeutung. Diese wird dann nach dem Umsatz und nicht nach der Betroffenheit von Unternehmen verschiedener Mitgliedstaaten bemessen: Sie soll vorliegen, wenn unter anderen ein gemeinschaftsweiter Gesamtumsatz von mindestens zwei beteiligten Unternehmen von jeweils mehr als 250 Mio. Euro gegeben ist; dies gilt nur dann nicht, wenn die beteiligten Unternehmen jeweils mehr als zwei Drittel ihres gemeinschaftsweiten Gesamtumsatzes in ein und demselben Mitgliedstaat erzielen (Art. 1 Abs. 2).

Ausschlaggebendes Kriterium ist damit der Schwellenwert des Umsatzes. Die Betroffenheit mehrerer Mitgliedstaaten erlangt erst auf einer zweiten, nachgeordneten Ebene Bedeutung, wenn nämlich die Schwellenwerte nicht erreicht werden. In diesem Fall soll dann gleichwohl eine gemeinschaftsweite Bedeutung bejaht werden können, wenn der Gesamtumsatz aller beteiligten Unternehmen in mindestens drei Mitgliedstaaten jeweils 100 Mio. Euro übersteigt (Art. 1 Abs. 3).

Diese Verteilung einer Zuständigkeit - die EU soll nur bei gemeinschaftsweiten Zusammenschlüssen, die oberhalb der Schwellenwerte liegen, zuständig sein - hat Calliess als Anwendungsfall der Subsidiarität im konservativen Sinne bezeichnet. ${ }^{76}$ Mithilfe der Schwellenwerte werde die zwischen Union und Mitgliedstaaten konkurrierende Kompetenz im Sinne einer Entweder-oder-Entscheidung aufgeteilt. ${ }^{77}$ Das Subsidiaritätsprinzip sei hier dadurch verwirklicht, dass eine Zuständigkeit eindeutig an eine der beiden Ebenen verwiesen werde. ${ }^{78}$

\section{Subsidiaritätsprinzip, Kontrollkompetenzen und grenzüberschreitender Sachverhalt}

\section{a) Der transnationale Bezug im Subsidiaritätsprinzip}

Die genannten Regeln der Fusionskontrolle verdeutlichen anschaulich den engen Zusammenhang, der zwischen dem Subsidiaritätsprinzip und dem grenzüberschreitenden Sachverhalt besteht. Man kann davon ausgehen, dass das Subsidiaritätsprinzip durch einen starken transnationalen Bezug gekennzeichnet ist.

Dies gilt schon für den Wortlaut der primärrechtlichen Verbürgung des Subsidiaritätsprinzips: ${ }^{79}$ Nach Art. 5 Abs. 3 EUV darf die Union in Bereichen, die nicht in ihre aus-

75 VO (EG) Nr. 139/2004 des Rates vom 20.1.2004 über die Kontrolle von Unternehmenszusammenschlüssen, ABl. L 24 v. 29.1.2004, S. 1.

76 Calliess, (Fn. 55), S. $221 \mathrm{ff}$.

77 Ibid., S. 208.

78 Ibid., S. 227.

79 Zur allgemeinen Definition des Begriffs der Subsidiarität: Calliess, in: Blickle/Hüglin/Wyduckel, Subsidiaritätsprinzip als rechtliches und politisches Ordnungsprinzip in Kirche, Staat und Gesellschaft, Rechtstheorie-Beiheft 20, 2002, S. 371. 
schließliche Zuständigkeit fallen, nur tätig werden, sofern und soweit die Ziele der in Betracht gezogenen Maßnahmen auf Ebene der Mitgliedstaaten nicht ausreichend verwirklicht und daher besser auf Unionsebene erreicht werden können. ${ }^{80}$ Es muss also eine Maßnahme gegeben sein, die den einzelnen Mitgliedstaat überfordern würde, ${ }^{81}$ also ein über den Hoheitsbereich eines Mitgliedstaats hinausgehendes Problem vorliegen: ${ }^{82}$

„Der betreffende Bereich weist transnationale Aspekte auf, die durch Maßnahmen der Mitgliedstaaten nicht ausreichend geregelt werden können. “63

In positiver Hinsicht ist bei der Frage, ob eine Maßnahme „wegen ihres Umfangs oder ihrer Wirkungen“ (Art. 5 Abs. 3 EUV) auf Unionsebene besser erreicht werden kann, ebenfalls die Frage des grenzüberschreitenden Bezugs zu stellen. Die Union kann naturgemäß grenzüberschreitende Problemlagen besser lösen als einzelne Mitgliedstaaten. ${ }^{84}$

In diesem Sinne stellt die Literatur transnationale Aspekte als Bewertungskriterium heraus. ${ }^{85}$ Dabei besteht aber Uneinigkeit darüber, ob nur qualitative oder auch quantitative Kriterien - wobei letztere vor allem in die Nähe von Effizienzerwägungen gestellt werden ${ }^{86}$ - zur Begründung des europäischen Mehrwerts herangezogen werden sollen. ${ }^{87}$ Zum Teil werden aber auch beide Kriterien zusammen herangezogen; in diesem Sinne will beispielsweise Calliess auf Größe und Schwere und auf die Frage, ob tatsächlich mehrere Mitgliedstaaten betroffen sind, abstellen. In diesem Sinne war wohl auch Nr. 4 des Subsidiaritätsprotokolls zu verstehen, der festhält, dass

„die Feststellung, dass ein Gemeinschaftsziel besser auf Gemeinschaftsebene erreicht werden kann, [...] auf qualitativen oder - soweit möglich - auf quantitativen Kriterien beruhen [muss]“.

80 Vgl. insbes. Calliess, (Fn. 55), S. 76 ff.; Calliess, Bitburger Gespräche, Jahrbuch 2011/I, 2012, S. $53 \mathrm{ff}$.

81 Schmidhuber/Hitzler, Die Verankerung des Subsidiaritätsprinzips im EWG-Vertrag - ein wichtiger Schritt auf dem Weg zu einer föderalen Verfassung der Europäischen Gemeinschaft, NVwZ 1992, S. 723.

82 Jarass, Grundfragen der innerstaatlichen Bedeutung des EG-Rechts, 1994, S. 18. Ausdrücklich auch Calliess, Bitburger Gespräche, (Fn. 80), S. 57.

83 Protokoll (Nr. 30) über die Anwendung der Grundsätze der Subsidiarität und der VerhältnismäBigkeit (1997), ABl. C 340 v. 10.11.1997, S. 105, Ziffer 5 (im Folgenden: Subsidiaritätsprotokoll). Diese Formulierung geht auf die Schlussfolgerungen des Rates von Edinburgh zurück, Europäischer Rat von Edinburgh v. 12.12.1992, Schlussfolgerungen des Vorsitzes, DOC/92/8, Anlage I, Gesamtkonzept für die Anwendung des Subsidiaritätsprinzip und des Artikels $3 \mathrm{~b}$ des Vertrags über die Europäische Union durch den Rat, II. Absatz 2, ii); abgedruckt in Calliess, (Fn. 55), S. $391 \mathrm{ff}$.

84 Calliess, Die neue Europäische Union nach dem Vertrag von Lissabon, 2010, S. 210. Auch Mitteilung der Kommission an den Rat und an das Europäische Parlament betreffend das Subsidiaritätsprinzip, SEK(92)1990 v. 27.11.1992, Anhang II, veröffentlicht in EUROPE/Dokumente Nr. 1804/05 v. 30.10.1992, abgedruckt auch in Bulletin EG 10, 1992, S. 125 und Merten, Die Subsidiarität Europas, 1993, S. 112.

85 Kingreen, Die Struktur der Grundfreiheiten des Europäischen Gemeinschaftsrechts, 1999, S. 111.

86 Vgl. Lienbacher, in: Schwarze, EU-Kommentar, 3. Aufl. 2012, Art. 5 EUV, Rdnr. 25, der das Positivkriterium ausdrücklich Effizienzkriterium nennt. Schmidhuber, Das Subsidiaritätsprinzip im Vertrag von Maastricht, DVBl. 1993, S. 419; von Borries, Das Subsidiaritätsprinzip im Recht der Europäischen Union, EuR 1994, S. 276 f.

87 Vgl. Calliess, Bitburger Gespräche, (Fn. 80), S. 57. Ablehnend Pieper, Subsidiaritätsprinzip Strukturprinzip der Europäischen Union, DVB1. 1993, S. 709. 
Der EuGH umschreibt in den wenigen Fällen, in denen er das Subsidiaritätsprinzip ausdrücklich geprüft hat, ${ }^{88}$ interessanterweise das Positivkriterium mit oben bereits im Rahmen der Rechtsprechung zu den Grundfreiheiten erörterten Kriterien. So begründet er die Notwendigkeit einer Biopatentrichtlinie damit, dass die Vorschriften das reibungslose Funktionieren des Binnenmarktes gewährleisten sollen; dazu stellt er ausdrücklich auf den „innergemeinschaftlichen Handel“ ab:

„Da das Ausmaß dieses Schutzes unmittelbare Auswirkungen [...] auf den innergemeinschaftlichen Handel hat, liegt es im Übrigen auf der Hand, dass das fragliche Ziel [...] besser auf Gemeinschaftsebene erreicht werden kann." ${ }^{\text {"89 }}$

\section{b) Kontrollkompetenzen}

Das Subsidiaritätsprinzip findet allerdings nur auf die Legislativkompetenzen der Union Anwendung, ${ }^{90}$ da nur diese abstrakt-generelle Maßnahmen darstellen. ${ }^{91}$ Der Begriff der Maßnahme in Art. 5 Abs. 3 EUV ist zu begrenzen auf alle im Wege der Gesetzgebung konkretisierenden Tätigkeiten der Union, die unmittelbar oder mittelbar Rechtswirkungen entfalten können. ${ }^{92}$ Das Subsidiaritätsprinzip begrenzt die positiv vorhandenen Unionskompetenzen im Sinne einer Feinsteuerung durch Zuordnung einzelner Maßnahmen zu verschiedenen Ebenen. ${ }^{93}$ Dies bedeutet, dass es auf die so genannten Kontrollkompetenzen, zu denen die Grundfreiheiten, die Kartellregeln, aber auch das unionsbürgerschaftliche Freizügigkeitsrecht gehören, ${ }^{94}$ nicht übertragen werden kann. ${ }^{95}$

88 Vgl. Colneric, Der Gerichtshof der Europäischen Gemeinschaften als Kompetenzgericht, EuZW 2002, S. 713.

89 EuGH, Rs. C-377/98, Biopatentrichtlinie, Slg. 2001, I-7079, Rdnr. 32.

90 Calliess, in: Calliess/Ruffert, (Fn. 4), Art. 5 EUV, Rdnr. 29 f.; Lienbacher, (Fn. 86), Art. 5 EUV, Rdnr. 21.

91 Unklar Streinz, in: Streinz, EUV/AEUV, 2. Aufl. 2012, Art. 5 EUV, Rdnr. 21, nach dem das Subsidiaritätsprinzip ,insbesondere“ für die Rechtsetzung, darüber hinaus aber auch für die „,gesamte Tätigkeit“" der Union gelten soll. Vgl. zur Diskussion auch Schlag, Grenzüberschreitende Verwaltungsbefugnisse im EG-Binnenmarkt, 1998, S. $145 \mathrm{ff}$.

92 Calliess, (Fn. 55), S. 101 ff., insbes. S. 103.

93 Europäischer Rat in Edinburgh, Tagung der Staats- und Regierungschefs der Europäischen Gemeinschaft am 11.-12.12.1992, Schlussfolgerungen des Vorsitzes, Teil A, Anlage 1, II. Leitlinien, Absatz 1 (Grenze für das Tätigwerden der Gemeinschaft), abgedruckt in Merten, (Fn. 84), S. 136; Trüe, Das System der Rechtsetzungskompetenzen der Europäischen Gemeinschaft und der Europäischen Union, 2002, S. 75 f.; Rengeling, Die Kompetenzen der Europäischen Union, Festschrift Badura, 2004, S. 1144.

94 Dazu ausführlich Lippert, (Fn. 12), S. 298 ff.

95 Calliess, (Fn. 90), Art. 5 AEUV, Rdnr. 28. A.A. Rohe, Binnenmarkt oder Interessenverband?, Zum Verhältnis von Binnenmarktziel und Subsidiaritätsprinzip nach dem Maastricht-Vertrag, RabelsZ 1997, Bd. 61, S. 1; wohl auch Ullmann, Die Europäische Union und das nationale Wettbewerbs- und Urheberrecht, JZ 1994, S. 929. 
Kontrollkompetenzen stellen neben den Legislativkompetenzen die zweite wesentliche Kompetenzart zur Verwirklichung des Binnenmarktes im Sinne von Art. 26 AEUV dar. Sie sind bislang nicht vertieft analysiert worden. ${ }^{96}$

Mit den Kontrollkompetenzen kann die Union Regelungen der Mitgliedstaaten bzw. das Verhalten privater Marktteilnehmer auf ihre Europarechts- und Binnenmarktkonformität hin kontrollieren. Anders als die Legislativkompetenzen dienen sie nicht dazu, einen speziellen sachlich-gegenständlichen Bereich - wie zum Beispiel die unionale Infrastrukturpolitik $^{97}$ - inhaltlich gestaltend zu regeln. Der Begriff der Kontrollkompetenz beschreibt vielmehr die Möglichkeit der Union, im Einzelfall anhand festgelegter Maßstäbe eine Kontrolle auszuüben.

In diesem Sinne sind sie auch repressiv ausgerichtet: Sie greifen erst dann, wenn auf Grundlage bereits bestehenden mitgliedstaatlichen Rechts eine nachträgliche Kontrolle zur Beseitigung von Binnenmarktbehinderungen ausgeübt werden soll. Die Legislativkompetenzen hingegen wirken präventiv, denn sie ermächtigen den Unionsgesetzgeber im Vorfeld zum Erlass von Regelungen zur Lösung eines Problems.

Deshalb entziehen sich die Kontrollkompetenzen auch der Zuordnung zu einer geschriebenen, positiven Kompetenzgrundlage. ${ }^{98}$ Sie lassen sich nicht wie die Legislativkompetenzen abschließend in einem Zuständigkeitskatalog zusammenstellen. So gelten beispielsweise die Grundfreiheiten auch und gerade dort, wo die Mitgliedstaaten ihre alleinige Kompetenz behalten haben. Denn die Kompetenzen im legislativen Bereich befreien nicht von den materiellen Bindungen an die Vorgaben. ${ }^{99}$ Dies erklärt dann auch, warum die Mitgliedstaaten beispielsweise im Bereich der sozialen Sicherheit, in denen sie weiterhin

96 Ausführliche Darstellung bei Lippert, (Fn. 12), S. 296 ff. Darüber hinaus findet sich der Begriff mit Ansätzen zu einer systematischen Standortbestimmung - soweit ersichtlich - nur bei Calliess, in: Calliess/Ruffert (Hrsg.), Verfassung der Europäischen Union, 2006, Art. I-13, Rdnr. 9; Calliess, (Fn. 90), Art. 3 AEUV, Rdnr. 9, Art. 5 EUV, Rdnr. 30; Calliess, Der Binnenmarkt, die europäische Kompetenzordnung und das Subsidiaritätsprinzip im Lichte der neuen Europäischen Verfassung, Festschrift Fischer, 2004, S. 22; Calliess, Kontrolle zentraler Kompetenzausübung in Deutschland und Europa: Ein Lehrstück für die Europäische Verfassung, Zugleich eine Besprechung des Altenpflegegesetz-Urteils des BVerfG, EuGRZ 2003, S. 181; Calliess, in: Brugger/ Kirste/Anderheiden, Gemeinwohl in Deutschland, Europa und der Welt, 2002, S. 181; Calliess, in: Bauer/Huber/Sommermann, Demokratie in Europa, 2005, S. 303. Nun auch Kingreen, Grundrechtsverbund oder Grundrechtsunion? - Zur Entwicklung der subjektiv-öffentlichen Rechte im europäischen Unionsrecht, EuR 2010, S. 356.

97 Vgl. dazu nur Calliess/Lippert, § 2, Transeuropäische Netze, in: Hatje/Müller-Graff (Hrsg.), Enzyklopädie Europarecht, Bd. 8, 2014.

98 Trüe, (Fn. 93), S. 160, 167. Ähnlich auch Müller-Graff, Systemrationalität in Kontinuität und Änderung des Europäischen Verfassungsvertrags, integration 2003, S. 306. Scharpf, Weshalb die EU nicht zur sozialen Marktwirtschaft werden kann, ZSE 2009, S. $423 \mathrm{f}$.

99 Kingreen, in: Calliess/Ruffert, (Fn. 4), Art. 34-36 AEUV, Rdnr. 17. Vgl. auch Pechstein, Die Mitgliedstaaten der EG als „Sachwalter des gemeinsamen Interesses“, 1987, S. 52. 
über die alleinige Rechtsetzungskompetenz verfügen, die Grundfreiheiten beachten müssen. ${ }^{100}$

Auch gewähren die Kontrollkompetenzen den Berechtigten subjektive Rechte. ${ }^{101}$ Dadurch wird der einzelne Unionsbürger in die Lage versetzt, unionsrechtlichen Vorgaben zur Durchsetzung zu verhelfen. ${ }^{102}$ So sind die Grundfreiheiten, das unionsbürgerschaftliche Freizügigkeitsrecht und die kartellrechtlichen Verbotsvorschriften unmittelbar anwendbar und der einzelne Unionsbürger wird durch sie unmittelbar berechtigt und verpflichtet; ${ }^{103}$ sie bedürfen keiner legislativen Ausgestaltung, um wirksam werden zu können. ${ }^{104}$

Kontrollkompetenzen sind typisch für föderale Mehrebenensysteme: Nur dort kann die eine über die andere Ebene Kontrolle ausüben. ${ }^{105}$ Dies setzt voraus, dass die untere Ebene, die kontrolliert werden soll, noch über Kompetenzen verfügt; denn nur in diesem Fall kann die untergeordnete Ebene Regelungen erlassen, die der Kontrolle der übergeordneten Ebene unterliegen. Deshalb können auf Kontrollkompetenzen auch Überlegungen zur Kompetenzverteilung von Legislativkompetenzen nicht übertragen werden. ${ }^{106}$ Dies gilt auch für das Subsidiaritätsprinzip, das im Sinne einer Kompetenzausübungsregel die Zuteilung von Sachmaterien zwischen zwei - prinzipiell gleichberechtigt nebeneinanderstehenden Ebenen vornehmen soll. Im Rahmen der Kontrollkompetenzen gibt es keine Sachmaterie, die entweder durch die eine (untergeordnete) oder durch die andere (übergeordnete) Ebene wahrgenommen werden könnte. ${ }^{107}$

100 EuGH, Rs. C-169/07, Hartlauer, Slg. 2009, I-1721, Rdnr. 29 unter Verweis auf EuGH, Rs. 238/82, Duphar u.a., Slg. 1984, 523, Rdnr. 16; EuGH, Rs. C-372/04, Watts, Slg. 2006, I-4325, Rdnrn. 92 und 146; EuGH, Rs. C-141/07, Slg. 2008, I-6935, Rdnr. 22 f.; EuGH, Rs. C-158/96, Kohll, Slg. 1998, I-1931, Rdnr. 19; EuGH, Rs. C-157/99, Smits und Peerbooms, Slg. 2001, I-5473, Rdnr. 46. Dazu Kanitz/Steinberg, Grenzenloses Gemeinschaftsrecht?, Die Rechtsprechung des EuGH zu Grundfreiheiten, Unionsbürgerschaft und Grundrechten als Kompetenzproblem, EuR 2003, S. 1013; Nowak, Zur grundfreiheitlichen Inanspruchnahme von Gesundheitsleistungen im europäischen Binnenmarkt, EuR 2003, S. 645; Cremer, Negative und positive Integration: Die Europäische Gemeinschaft als Sozial- und Bildungsunion, EuR Beiheft 1/2007, S. 77. Instruktiv Kingreen, Das Sozialstaatsprinzip im europäischen Verfassungsverbund, 2003, S. 354 ff.; auch Dettling, Ethisches Leitbild und EuGH-Kompetenz für die Gesundheitssysteme?, Zugleich Anmerkung zum Urteil des EuGH vom 16.5.2006 (Watts), EuZW 2006, S. 519; Wollenschläger, Patientenmobilität in der Europäischen Union - von der Rechtsprechung des EuGH zur neuen Richtlinie 2011/24/EU über die Ausübung der Patientenrechte in der grenzüberschreitenden Gesundheitsversorgung, EuR 2012, S. 155.

101 Mestmäcker/Schweitzer, (Fn. 4), § 2, Rdnr. 30 ff.

102 EuGH, Rs. 106/77, Slg. 1978, 629, Rdnr. 17/18; Frenz, (Fn. 10), Rdnr. 93; Calliess, Grundlagen, Grenzen und Perspektiven europäischen Richterrechts, NJW 2005, S. 931.

103 Vgl. für letztere Art. 1 VO (EG) Nr. 1/03. Vgl. auch Schubert, Der Gemeinsame Markt als Rechtsbegriff - Die allgemeine Wirtschaftsfreiheit des EG-Vertrages, 1999, S. $133 \mathrm{ff}$., $216 \mathrm{ff}$.

104 Calliess, in: Calliess/Ruffert, EUV/EGV, 3. Aufl. 2007, Art. 5 EGV, Rdnr. 26; Calliess, in: Calliess/Ruffert, (Fn. 96), Art. I-13, Rdnr. 9.

105 Aufgrund des Vorrang des Unionsrechts in den Bereichen, in denen die Union zur Rechtsetzung befugt ist, kann auch von einer Überordnung der Union über die Mitgliedstaaten gesprochen werden, vgl. Oppermann/Classen/Nettesheim, (Fn. 10), § 4, Rdnr. 9. Anders wohl Kirchhof, in: von Bogdandy/Bast, (Fn. 44), S. 1023, der eine Überordnung wohl grundsätzlich verneint.

106 Vgl. die Beschreibung des Problems bei von Bogdandy/Bitter, Unionsbürgerschaft und Diskriminierungsverbot, Festschrift Zuleeg, 2005, S. 317.

107 Vgl. zum ,entweder - oder“ als Ausdruck der sog. Subsidiarität im konservativen Sinne Calliess, (Fn. 55), S. 208. 


\section{c) Der grenzüberschreitende Sachverhalt bei den Kontrollkompetenzen}

Gleichwohl muss der Gedanke des grenzüberschreitenden Bezugs auch bei den Kontrollkompetenzen verwirklicht werden. Dies geschieht - um das Ergebnis vorwegzunehmen durch eine stärkere Konturierung des grenzüberschreitenden Sachverhalts als Tatbestandsmerkmal der Kontrollkompetenzen. ${ }^{108}$ Es übernimmt dann im Rahmen der Kontrollkompetenzen die Funktion des Subsidiaritätsprinzips bei den Legislativkompetenzen. Wenn bei Letzteren das Subsidiaritätsprinzip als Kompetenzausübungsregel ausgemacht wurde, dieses Prinzip aber auf die Kontrollkompetenzen nicht übertragen werden kann, dann bedarf es im Rahmen der Kontrollkompetenzen auch eines Kriteriums, das die Tätigkeit der Union auf die tatsächlich grenzüberschreitenden Problemlagen beschränkt. Denn nur in diesen Fällen soll die Kontrolle der übergeordneten, europäischen Ebene tatsächlich überhaupt erst aktiviert werden. Die Union soll nach dem Willen der Mitgliedstaaten ${ }^{109}$ nur dann Maßnahmen auf ihre Europarechtskonformität überprüfen, wenn ein grenzüberschreitendes Problem vorliegt.

Nur durch die Betonung des grenzüberschreitenden Bezugs bei der Anwendung der Kontrollkompetenzen kann verhindert werden, dass diese ihre repressive Ausrichtung, die sie auf die nachträgliche Kontrolle einer bereits getroffenen Entscheidung ${ }^{110}$ beschränkt, verlieren und auf präventive, gestalterische Tätigkeiten ausgedehnt werden. Dies würde nämlich die Grenze zu den Legislativkompetenzen verschwimmen lassen. ${ }^{111}$

Insofern sind die oben aufgeführten Kriterien zur Umschreibung des grenzüberschreitenden Sachverhalts, die eine Prognose und eine quantitative Abschätzung notwendig machen, problematisch. Kontrollkompetenzen sind nur darauf gerichtet, konkrete Maßnahmen nachträglich zu überprüfen, nicht aber positiv Recht zu setzen. ${ }^{112}$ Durch prognostische, quantitative Elemente jedoch wird die Prüfung vom konkreten Fall weggelenkt und es werden verallgemeinerbare Aussagen getroffen. So ist die Frage, ob eine mitgliedstaatliche Maßnahme geeignet und verhältnismäßig ist, den innergemeinschaftlichen Handel zu beschränken, von der konkreten Fallkonstellation losgelöst und auf generelle Aussagekraft gerichtet. $^{113}$

Dies widerspräche dann aber nicht nur der Kompetenzverteilung innerhalb der Union, sondern verkennt auch die Funktionsweise der beiden Kompetenzarten. Bei den Legislativkompetenzen nämlich hat die Prüfung, ob die Union aus Gründen der besseren Problemlösungsfähigkeit die Kompetenz zur Regelung eines Sachbereichs erhalten soll - nach den obigen Ausführungen wird dies in der Regel dann der Fall sein, wenn eine grenzüberschreitende Problemlage vorliegt - bereits stattgefunden, als die vertragsschließenden Par-

108 Vgl. Nettesheim, (Fn. 44), S. $343 \mathrm{f}$.

109 Dazu ausführlich Lippert, (Fn. 12), S. 231 ff.

110 Waldhoff, Verfassungsfragen der Kontrollkompetenzen des Landesrechnungshofs in gestuften Vermögensprivatisierungen, NWVB1. 2009, S. 371; ebenso Scheuner, Verantwortung und Kontrolle im demokratischen Rechtsstaat, Festschrift Müller, 1970, S. 379.

111 Vgl. Frenz, (Fn. 10), Rdnrn. 28 f., 31 f.

112 Vgl. Trüe, (Fn. 93), S. 159.

113 Kingreen, (Fn. 85), S. 131. Vgl. auch Adrian, Juristische Methodenlehre und die Keck-Entscheidung des EuGH, EWS 1998, S. 290. Ausführlich, auch zum Kriterium der VerhältnismäBigkeit Lippert, (Fn. 12), S. 358. 
teien, die Mitgliedstaaten, sich entschieden haben, der Union eine solche Kompetenz zu übertragen. Man kann also davon ausgehen, dass die Union nur diejenigen Kompetenzen übertragen bekommen hat, für deren Erfüllung sie die Mitgliedstaaten als besser geeignet angesehen haben. ${ }^{114}$

Bei den Kontrollkompetenzen aber ist eine solche Eignungsprüfung im Vorfeld nicht möglich. Sie können nämlich nicht - wie gezeigt - positiv in einem Katalog zusammengefasst werden. Um aber auch hier sicherzustellen, dass die Union befugt ist, die Kompetenz im jeweiligen Fall auszuüben, muss ihre Aktivierung durch das zusätzliche Merkmal des grenzüberschreitenden Sachverhalts gesteuert werden. Der grenzüberschreitende Sachverhalt ist, wird er denn als Kriterium ernstgenommen und inhaltlich mit Leben gefüllt, im Rahmen der Kontrollkompetenzen das Eignungskriterium, dessen Prüfung bei den Legislativkompetenzen schon im Zeitpunkt der Konstituierung vorweggenommen wurde. Durch ihn wird verhindert, dass die Kontrollkompetenzen systemwidrig zu Kompetenzen mit legislativer Gestaltungskraft werden.

Wie aber kann ein solchermaßen ernstgenommener, inhaltlich konturierter grenzüberschreitender Bezug aussehen? Diese Frage ist nicht leicht zu beantworten, wenn man dem oben erörterten, von der Rechtsprechung verwendeten und als quantitativ und nach ökonomischen Parametern ausgerichtete Definitionsansatz nicht folgen will.

Die Entwicklung einer Alternative setzt voraus, dass man sich der ursprünglichen Funktion und dem intendierten Zweck des grenzüberschreitenden Sachverhalts als Tatbestandsmerkmal der Kontrollkompetenzen besinnt. Dieser besteht - wie schon mehrfach angeklungen ist - darin, dass die Union ihre Tätigkeit aus Sicht der Mitgliedstaaten nur dann entfalten soll, wenn grenzüberschreitende Problemlagen gegeben sind, die einzelne Mitgliedstaaten überfordern. Die Mitgliedstaaten wollten und wollen die Union nicht etwa an ihre Stelle setzen, sondern sich ihrer nur bedienen, um bestimmte Probleme besser lösen zu können. Die souveräne Staatlichkeit der Mitgliedstaaten sollte auch im Verbund mit der Union erhalten bleiben - auch wenn sie inhaltlich eine weitgehende Veränderung erfährt und erfahren hat, sodass wohl heute von einer zwischen Union und Mitgliedstaaten geteilten Souveränität ausgegangen werden muss. ${ }^{115}$ Genau dies muss sich dann aber auch im Kriterium des grenzüberschreitenden Sachverhalts widerspiegeln.

\section{Die Alternative: Ein qualitatives Kriterium}

\section{Ein rechtlicher Maßstab}

Soll der grenzüberschreitende Sachverhalt auch das Verhältnis von Mitgliedstaaten und Union in rechtlicher Hinsicht widerspiegeln, muss er als Tatbestandsmerkmal der Kontrollkompetenzen nach rechtlichen Kriterien ausgelegt werden. ${ }^{116}$ Denn die fortbestehende

114 Trüe, (Fn. 93), S. 63. Stettner, Grundfragen einer Kompetenzlehre, 1983, S. 205 f., 308 ff. Das Subsidiaritätsprinzip bezieht sich auf die Kompetenzwahrnehmung, nicht auf die Kompetenzverteilung, Calliess, (Fn. 90), Art. 5 EUV, Rdnr. 2.

115 Vgl. dazu ausführlich Lippert, (Fn. 12), S. 248.

116 A.A. Papadileris, (Fn. 1), S. 127 f., nach der an die Stelle des grenzüberschreitenden Bezugs ein „konkreter, nachvollziehbarer und wirtschaftlicher Binnenmarktbezug“ treten soll. 
Regelungskompetenz der Mitgliedstaaten in der Europäischen Union lässt sich nur mit rechtlichen Kategorien erfassen; sie darf insbesondere auch nicht durch wirtschaftliche Kriterien - wie den Binnenmarkt - überdeckt werden.

Denn Grenzen sind in der Europäischen Union nur noch im rechtlichen Bereich zu finden - das gilt insbesondere nach Wegfall aller physischen Grenzbarrieren durch das SchengenAbkommen (vgl. jetzt Art. 77 AEUV). Di Fabio spricht insofern zutreffend von „Rechtszuordnungsgrenzen“. Diese Bedeutung sei den Grenzen in Europa noch verblieben, wenn der Binnenmarkt zu einem Bedeutungsverlust der sichtbaren und der wirtschaftlichen Grenze führe:
„Solange die alten Nationen Europas ihren Selbstgestaltungsanspruch nicht aufgeben, bleiben Grenzen als Staatsgrenzen wirksam, obwohl sie für den Bürger unsichtbar werden. Es tritt eine Juridifizierung der Grenze ein, so wie in anderen Epochen eine Militarisierung des Grenzraums zu beobachten war." 117

Dass im Zuge der europäischen Integration der Eindruck entstand, der vollendete Binnenmarkt verlange auch eine Negierung der Rechtszuordnungsgrenzen und damit der mitgliedstaatlichen Regelungsgewalt, liegt an der Verbindung von Markt und „Staat“, die sich so nur innerhalb der Europäischen Union findet. Denn ursprünglich waren nationalstaatliche Regulierungsgewalt und Marktorganisation deckungsgleich: Märkte waren vor allem nationale Märkte. Dies änderte sich aber in dem Augenblick, in dem mit der EU eine weitere Hoheitsgewalt auf den Spielplan trat, die nicht mehr nationalstaatlich organisiert war. ${ }^{118}$ Sie konnte und kann Märkte regulieren, ohne an staatliche Grenzen gebunden zu sein. Sie sollte dies im Übrigen auch ganz bewusst, denn die politische Integration wurde in bewusster Abkehr von politischen Festlegungen gerade auf eine wirtschaftliche Integration ausgerichtet: Die Friedensordnung sollte durch den Gemeinsamen Markt hergestellt werden, der Markt wurde zum zentralen Instrument der gemeinschaftlichen Zielsetzung. ${ }^{119}$ Recht und Wirtschaft gingen also im Zuge der europäischen Integration eine enge Verbindung ein: ${ }^{120}$ Mit der zunehmenden wirtschaftlichen Verflechtung der mitgliedstaatlichen Märkte verlor auch der tatsächliche Grenzübertritt an Bedeutung und an seine Stelle trat eine auf den Binnenmarkt bezogene Gesamtbetrachtung. Dies führte nicht nur zu der verbreiteten Einschätzung, die Grenzen verlören im Binnenmarkt ihre Bedeutung, ${ }^{121}$ sondern erklärt auch die Verwendung des Terminus der „Auswirkungen auf den innergemeinschaftlichen Handel“", den der EuGH zur Umschreibung des grenzüberschreitenden Sachverhalts verwendet.

Für einen Definitionsversuch des grenzüberschreitenden Sachverhalts darf jedoch nicht verkannt werden, dass allein die Tatsache der wirtschaftlichen Verflechtung noch keine Aussage über die Beurteilung eines Sachverhalts in rechtlicher Hinsicht zu treffen ver-

117 Di Fabio, Der Verfassungsstaat in der Weltgesellschaft, 2001, S. 67. Explizit auf einen rechtlichen Ansatz im Rahmen des grenzüberschreitenden Elements im Europarecht stellt auch Lach, (Fn. 15), S. 248 ab.

118 Wagener/Eger/Fritz, Europäische Integration, 2006, S. 24.

119 Haltern, in: von Bogdandy/Bast, (Fn. 44), S. 308, 313. Vgl. auch Schulze/Walter, in: Schulze/ Walter, 50 Jahre Römische Verträge, 2008, S. 10.

120 Ausführlich Lippert, (Fn. 12), S. 369 ff.

121 Vgl. Roth, (Fn. 33), S. 584. 
mag. ${ }^{122}$ Es trifft zwar zu, dass wegen der hohen Verflechtungsdichte auf dem Binnenmarkt jede nationale Maßnahme auch grenzüberschreitende Auswirkungen hat. Dies sagt aber noch nichts darüber aus, welches Recht Anwendung finden soll. Alleine das Vorliegen wirtschaftlicher Verflechtungen kann nicht zur Anwendbarkeit von Unionsrecht führen. ${ }^{123}$ Genau dies verbirgt sich aber hinter der Gleichsetzung von grenzüberschreitendem Sachverhalt und den Auswirkungen auf den innergemeinschaftlichen Handel: Da jede Wirtschaftstätigkeit immer auch Auswirkungen auf den innergemeinschaftlichen Handel hat, muss ein derart definierter transnationaler Bezug immer vorliegen. Dadurch wäre nach dieser Definition fast immer Unionsrecht anwendbar. Ein solcher Maßen verstandener grenzüberschreitender Sachverhalt würde aber keine wesentliche Funktion mehr entfalten können. Dies kann er nur dann, wenn er deutlich unterscheidet zwischen einerseits dem Ziel, in wirtschaftlicher Hinsicht einen einheitlichen Markt zu schaffen und andererseits der Erkenntnis, dass dadurch in rechtlicher Hinsicht staatliche Regelungsgrenzen nicht negiert werden können.

\section{Eine neue Definition des grenzüberschreitenden Sachverhalts}

Soll also das Kriterium des grenzüberschreitenden Sachverhalts - plakativ zusammengefasst - den Fortbestand der mitgliedstaatlichen Regelungszuständigkeiten im Binnenmarkt bzw. trotz des Binnenmarkts abbilden, ${ }^{124}$ dann liegt es nahe, dieses Kriterium an den traditionellen Merkmalen von Staatlichkeit zu orientieren; nach der klassischen DreiElemente-Lehre sind dies Staatsgebiet, Staatsgewalt und Staatsvolk. ${ }^{125}$

Die würde bedeuten, dass sich

(1.) aus dem Merkmal des Staatsgebiets das Erfordernis des tatsächlichen Grenzübertritts ergibt;

(2.) aus dem Merkmal der Staatsgewalt ergibt, dass ein Produkt oder eine Person zwei verschiedenen Rechtsordnungen unterworfen ist;

(3.) aus dem Merkmal des Staatsvolkes ergibt, dass ein grenzüberschreitender Sachverhalt nur dann vorliegt, wenn sich ein Staatsangehöriger gegenüber einem anderen Mitgliedstaat auf seine durch die Unionsrechtsordnung zur Verfügung gestellten Rechte beruft.

Das erste Merkmal des tatsächlichen Grenzübertritts schließt hypothetische oder nur möglicherweise in der Zukunft stattfindende Fallgestaltungen aus. ${ }^{126}$ Es vermeidet auch, dass allein Auswirkungen auf den gemeinsamen, innergemeinschaftlichen Markt ausreichen, um von einer grenzüberschreitenden Gefährdungslage sprechen zu können. ${ }^{127}$

Zusätzlich muss das betreffende Produkt bzw. die betreffende Person gleichzeitig zwei Rechtsordnungen unterworfen sein. Denn würde nur der tatsächliche Grenzübertritt ausreichen, dann sind Fälle denkbar, in denen sich der Unionsbürger gegenüber seinem eigenen

122 A.A. Papadileris, (Fn. 1), S. $127 \mathrm{f}$.

123 Vgl. Mayer, (Fn. 23), S. 803.

124 Vgl. dazu auch die Ausführungen des BVerfG im Lissabon-Urteil, NJW 2009, 2267, Rdnr. 298.

125 Siehe dazu auch oben. Vgl. hier nur Jellinek, Allgemeine Staatslehre, 3. Aufl. 1920, S. 394 ff.

126 Vgl. auch Potacs, (Fn. 31), S. 481 f.

127 Lippert, (Fn. 12), S. 394. 
Mitgliedstaat auf die Grundfreiheiten beruft. Richtigerweise liegt aber in diesen Fällen gar kein grenzüberschreitender Sachverhalt vor; es handelt sich vielmehr um eine Regelung, die sowohl inländische als auch ausländische Anbieter betrifft - und damit im Sinne der „Keck“-Rechtsprechung eine Verkaufsmodalität ist, die nicht an den Grundfreiheiten gemessen werden soll. ${ }^{128}$

Das gleichzeitige Unterfallen des zu beurteilenden Sachverhalts unter zwei Rechtsordnungen bedeutet für den Betroffenen, dass er nicht nur die rechtlichen Voraussetzungen seines Herkunftsstaats, sondern auch diejenigen des Zielstaats beachten muss; ihm wird also die Beachtung zweier Rechtsregime zugemutet. ${ }^{129}$ Die Benachteiligung liegt in der Verdopplung der Anforderungen an den grenzüberschreitend Tätigen. Dagegen hilft insbesondere das aus den Grundfreiheiten bekannte Herkunftslandprinzip: Erfüllt der grenzüberschreitend Tätige alle Voraussetzungen in seinem Heimatstaat, dann können ihm unter der Geltung des Herkunftslandprinzips diese Voraussetzungen im Zielstaat nicht erneut abverlangt werden. ${ }^{130}$

Bei den Personenverkehrsfreiheiten, also Niederlassungs- und Arbeitnehmerfreizügigkeit sowie Art. 21 AEUV, kommt schließlich noch hinzu, dass die betroffene Person sich nicht gegenüber ihrem eigenen Mitgliedstaat auf ihre Rechte berufen darf. Sie muss also über eine andere Staatsangehörigkeit verfügen. ${ }^{131}$ Im Übrigen erfüllt diese Voraussetzung auch in dogmatischer Hinsicht eine wichtige Funktion: Sie ermöglicht nämlich eine Unterscheidung zwischen Grundfreiheiten und den Grundrechten. ${ }^{132}$

\section{Fazit: Die Bedeutung des grenzüberschreitenden Sachverhalts im Unionsrecht}

Seit jeher wird versucht, das wahre Wesen des Yetis zu ergründen. Ein Umstand, der für den grenzüberschreitenden Sachverhalt bislang leider nicht in gleichem Maße gilt - was verwunderlich ist, denn das Erkenntnisinteresse dürfte hier mindestens genauso groß wie seine Existenz unstreitig sein.

Für das Unionsrecht erfüllt das Merkmal des grenzüberschreitenden Bezugs eine außerordentlich wichtige Funktion. Es ist nicht nur der Schlüsselbegriff für die Kompetenzverteilung zwischen Union und Mitgliedstaaten; es kann auch für die Akzeptanz und die Legitimität europäischer Entscheidungen kaum überschätzt werden.

Der grenzüberschreitende Sachverhalt konzentriert Entwicklung, Stand und Eigenheit der europäischen Integration wie in einem Brennglas. Dies gilt insbesondere für die Entwicklung des Staaten- und Verfassungsverbundes, der auch als Werteverbund beschrieben wird: Die Union richtet sich zunehmend an außerökonomischen Werten und Zielen aus und

128 EuGH, Rs. C-267/91 und C-268/91, Keck und Mithouard, Slg. 1994, I-6097. Ausführlich Lippert, (Fn. 12), S. $401 \mathrm{f}$.

129 Kingreen, (Fn. 85), S. 60. Anklänge in diese Richtung bzgl. der Niederlassungsfreiheit auch in EuGH, Rs. C-378/10, VALE, noch nicht in der amtl. Slg., Rdnr. 37.

130 Vgl. Kingreen, (Fn. 85), S. 59 f.

131 Vgl. Jarass, Elemente einer Dogmatik der Grundfreiheiten, EuR 1995, S. 204 ff.

132 Vgl. dazu ausführlich Lippert, (Fn. 12), S. 404 f. 
sucht ihre Identität und ihren Telos nicht mehr nur in einer rein wirtschaftlich basierten Integration. ${ }^{133}$

In diesem Zusammenhang wird häufig auch die Rolle des EuGH kritisch hinterfragt. So wird in der Literatur zum Teil konstatiert, dass beispielsweise die Dogmatik der Grundfreiheiten und die grundfreiheitliche Rechtsprechung des EuGH den Anschluss an die verfassungsrechtliche Entwicklung der Union noch nicht gefunden hätten. ${ }^{134}$ Dies gilt auch für das Merkmal des grenzüberschreitenden Sachverhalts. Die Auslegung des im Rahmen der Europäischen Union als Rechtsgemeinschaft erlassenen Rechts darf sich nicht nur an wirtschaftlichen Parametern orientieren, sondern muss sich ihrer normativen Fundierung bewusst werden. Der Gerichtshof steht nicht mehr allein im Dienst einer möglichst weitgehenden wirtschaftlichen Integration; er ist vielmehr zunehmend zum Sachwalter eines spezifisch europäischen Gemeinwohls geworden, das sich gerade im Wege der Anwendung von Kontrollkompetenzen durch die Abwägung zwischen mitgliedstaatlichen und europäischen Interessenpositionen bildet. ${ }^{135}$

Voraussetzung für werteorientierte Entscheidungen in diesem Sinne ist ihre Legitimität. ${ }^{136}$ Der Entstehung von Gemeinwohl vorausgelagert ist die Frage der Zuständigkeit und der Kompetenz für die Entwicklung wertgebundener Antworten auf gesellschaftliche Fragen. ${ }^{137}$ Dabei bedarf es für ein funktionierendes politisches Gemeinwesen sowohl formeller Legitimität im Sinne rechtsstaatlicher Verfahren als auch materieller Legitimität im Sinne inhaltlicher Akzeptanz. ${ }^{138}$

Wenn die Rechtsprechung den bereits eingeschlagenen Weg einer nicht nur ökonomisch ausgerichteten, sondern auch werte- und gemeinwohlbezogenen Union mitgehen will, dann muss sie die Bedeutung, die einer klaren Kompetenzzuteilung und -verteilung im Rahmen der Kontrollkompetenzen zukommt, berücksichtigen. Nur eine klare, transparente und

133 Vgl. ausführlich zur Entwicklung und primärrechtlichen Verankerung Calliess, Europa als Wertegemeinschaft - Integration und Identität durch europäisches Verfassungsrecht?, JZ 2004, S. 1033. Auch Rensmann, in: Blumenwitz/Gornig/Murswiek, Die Europäische Union als Wertegemeinschaft, 2005, S. $50 \mathrm{ff}$.

134 Kingreen, (Fn. 44), S. 725. Vgl. auch Haltern, (Fn. 119), S. 309 ff. Ähnlich Schulze/Walter, (Fn. 119), S. 9 f.

135 Vgl. Uerpmann, Das öffentliche Interesse, 1999, S. 263 ff. Ähnlich, wenn auch mehr auf die Sensibilität zur Kompetenzabgrenzung zwischen Union und Mitgliedstaaten abstellend Calliess, Bitburger Gespräche, (Fn. 80), S. 79.

136 Stettner, (Fn. 114), S. 72.

137 Ibid., S. $203 \mathrm{ff}$.

138 Vgl. auch Brugger, Gemeinwohl als Ziel von Staat und Recht, Festschrift Quaritsch, 1990, S. 59 f., 63. Calliess, in: Brugger/Kirste/Anderheiden, (Fn. 96), S. 175. 
nachvollziehbare Aufteilung kann die Legitimität gerade in dem aus mehreren Ebenen bestehenden Staaten-, Verfassungs- und Gemeinwohlverbund sichern. ${ }^{139}$

Die Akzentuierung des grenzüberschreitenden Bezugs im Unionsrecht zielt darauf $a b$, Verantwortlichkeiten und Zuständigkeiten transparenter zu machen, um damit im Ergebnis dem Ziel einer spezifisch europäischen Demokratisierung und Identitäts- und Gemeinwohlfindung $\mathrm{zu}$ dienen. ${ }^{140}$ Transparente Verantwortlichkeiten, klare Zuständigkeiten, Nachvollziehbarkeit und Rechtssicherheit sind notwendige Merkmale von Demokratie und Rechtsstaatlichkeit - zwei „grundlegende Werte“ ${ }^{141}$ zu denen sich die Europäische Union in Art. 2 EUV bekennt. Sie sind nicht nur wesentliche Voraussetzung für ihre Funktionsfähigkeit, sondern auch unabdingbar für die Akzeptanz und die Legitimität europäischer Entscheidungen.

139 Vgl. ibid., S. 204. Zur Verbindung von „abgrenzbarer politischer Entscheidungsgewalt“ und föderalen Systemen: Badura, in: Gesellschaft für Rechtspolitik Trier, Bitburger Gespräche 1999/ II, 50 Jahre Grundgesetz, 50 Jahre Föderalismus, 2000, S. 55. Vgl. zum Zusammenhang von Verantwortungsklarheit und Legitimation auch Trute, in: Hoffmann-Riem/Schmidt-Aßmann/ Voßkuhle, Grundlagen des Verwaltungsrechts, Bd. I, Methoden, Maßstäbe, Aufgaben, Organisation, 2. Aufl. 2012, § 6, Rdnr. 109. Auch Hatje, Demokratische Kosten souveräner Staatlichkeit im europäischen Verfassungsverbund - zu den Perspektiven der Demokratie in der Europäischen Union nach dem Urteil des Bundesverfassungsgerichts zum Vertrag von Lissabon, EuR-Beiheft 1/2010, S. 130 f. Vgl. auch Stettner, (Fn. 114), S. $260 \mathrm{ff}$.

140 Ähnlich Calliess, Bitburger Gespräche, (Fn. 80), S. 77 f. Zur Demokratisierung Hatje, (Fn. 139), S. $130 \mathrm{f}$

141 So die nichtamtliche Überschrift des Art. 2 EUV. 